\title{
Cloud-Radiation Feedback and Atmosphere-Ocean Coupling in a Stochastic Multicloud Model
}

\author{
Yevgeniy Frenkel \\ Department of Mathematics and Center for Atmosphere-Ocean Science, Courant Institute \\ New York University \\ 251 Mercer Street \\ New York, NY 10012 USA \\ Andrew J. Majda \\ Department of Mathematics and Center for Atmosphere-Ocean Science, Courant Institute, \\ and Center for Prototype Climate Modeling, NYU Abu Dhabi Institute \\ New York University \\ 251 Mercer Street \\ New York, NY 10012 USA \\ Samuel N. Stechmann* \\ Department of Mathematics and Department of Atmospheric and Oceanic Sciences \\ University of Wisconsin-Madison \\ 480 Lincoln $\mathrm{Dr}$ \\ Madison, WI 53706
}

\begin{abstract}
Despite recent advances in supercomputing, current general circulation models (GCMs) have significant problems in representing the variability associated with organized tropical convection. Furthermore, due to high sensitivity of the simulations to the cloud radiation feedback, the tropical convection remains a major source of uncertainty in long-term weather and climate forecasts. In a series of recent studies, it has been shown, in paradigm two-baroclinic-mode systems and in aquaplanet GCMs, that a stochastic multicloud convective parameterization based on three cloud types (congestus, deep and stratiform) can be used to improve the variability and the dynamical structure of tropical convection, including intermittent coherent structures such as synoptic and mesoscale convective
\end{abstract}

\footnotetext{
* Corresponding author

Email address: stechmann@wisc.edu (Samuel N. Stechmann )
}

Preprint submitted to Dynamics of Atmospheres and Oceans

May 8, 2015

(C) 2015. This manuscript version is made available under the Elsevier user license http://www.elsevier.com/open-access/userlicense/1.0/ 
systems. Here, the stochastic multicloud model is modified with a parameterized cloud radiation feedback mechanism and atmosphere-ocean coupling. The radiative convective feedback mechanism is shown to increase the mean and variability of the Walker circulation. The corresponding intensification of the circulation is associated with propagating synoptic scale systems originating inside of the enhanced sea surface temperature area. In column simulations, the atmosphere ocean coupling introduces pronounced low frequency convective features on the time scale associated with the depth of the mixed ocean layer. However, in the presence of the gravity wave mixing of spatially extended simulations, these features are not as prominent. This highlights the deficiency of the column model approach at predicting the behavior of multiscale spatially extended systems. Overall, the study develops a systematic framework for incorporating parameterized radiative cloud feedback and ocean coupling which may be used to improve representation of intraseasonal and seasonal variability in GCMs.

Keywords: Stochastic convective parameterization, multicloud models, tropical atmospheric dynamics, convectively coupled waves, cloud radiation feedback, atmosphere ocean coupling

\section{Introduction}

Atmospheric dynamics in the tropics are characterized by the predominance of organized convection on a wide range of scales, spanning mesoscale systems to synoptic and planetary-scale convectively coupled waves such as Kelvin waves 5 and the Madden Julian oscillation (MJO) (Nakazawa, 1974; Hendon and Liebmann, 1994; Wheeler and Kiladis, 1999). While the importance of the tropics to weather and climate forecasts cannot be overestimated, present coarse resolution GCMs used for the prediction of weather and climate have significant problems in representing variability associated with tropical convection (Slingo et al., 1996; Moncrieff and Klinker, 1997; Scinocca and McFarlane, 2004; Lau and Waliser, 2005; Zhang, 2005). 
It is believed that the deficiency is due to insufficient treatment of the cumulus convection (Moncrieff and Klinker, 1997; Lin et al., 2006), which has to be parameterized in GCMs. The inaccuracy of the vertical and horizontal cloud distributions furthers the already great uncertainty associated with cloud radiation feedback (CRF) mechanisms (Stephens and Webster, 1979; Cess et al., 1990, 1996; Bony and Emanuel, 2005) and the large-scale atmospheric circulation (Peters and Bretherton, 2005; Tian and Ramanathan, 2003). Given the sensitivity of tropical variability (Stephens and Webster, 1979; Cess et al., 1990, 1996; Bony and Emanuel, 2005) to CRF, the search for new strategies for the parameterization of tropical convection and associated radiative feedback and atmosphere ocean coupling (AOC) effects is one of the central problems in the atmospheric community.

Several methods have been developed to address the multiscale nature of tropical convection. Cloud-resolving models (CRM) on fine computational grids have succeeded in representing some aspects of organized convection, including mesoscale organization and cloud distribution (ECMWF, 2003; Moncrieff et al., 2007; Slawinska et al., 2014b). In addition, superparameterization (SP) methods (Grabowski and Smolarkiewicz, 1999; Grabowski, 2001, 2004; Randall so et al., 2003; Majda, 2007) and sparse space-time SP (Xing et al., 2009; Slawinska et al., 2014a) use a cloud resolving model in each column of the large scale GCM. In the SP setup, the CRM is used to explicitly represent small scale processes such as cloud microphysics and convective updrafts, thereby representing an extended range of the scales of tropical convection compared to a traditional GCM. However, despite considerable success in duplicating observed radiative fluxes (Wu and Moncrief, 2000), these methods are not currently computationally viable for application to climate simulations. Moreover, these complex models do not necessarily further the qualitative understanding of the processes involved. For example, CRF is usually computed through highly com40 plex multiple-scattering and radiative transfer models (Stephens and Webster, 1979; Zurovac-Jevtik et al., 2005).

Another novel approach to the problem of missing tropical variability in 
GCMs has been the development of the multicloud parameterizations (Khouider and Majda, 2006b,a, 2007, 2008a,b; Khouider et al., 2010; Frenkel et al., 2012; Peters et al., 2013), which capture the interaction of the three cloud types (congestus, deep and stratiform) which characterize tropical convection. In particular, the stochastic multicloud model (Khouider et al., 2010; Frenkel et al., 2012, 2013) (hereafter KBM10, FMK12, FMK13) aims to capture these phenomena with a Markov chain lattice model where each lattice site is either occupied by a cloud of a certain type or is considered a clear-sky site. The convective elements interact with the large-scale environment and with each other through convective available potential energy (CAPE) and middle troposphere dryness. When local interactions between the individual lattice sites are ignored, or when nearest-neighbor interactions are allowed (Khouider, 2014), the dynamical evolution of the cloud area fractions in the stochastic multicloud model takes the form of a computationally inexpensive coarse grained stochastic process (Katsoulakis et al., 2003; Khouider et al., 2003; Majda et al., 2008). In addition to an enhanced representation of clouds, the framework is simple enough to allow semi-analytic solutions. In particular these authors were able to study stability and bifurcations of the solutions attributable to the diurnal surface fluxes (Frenkel et al., 2010). Despite its apparent simplicity, the multicloud model is very successful in capturing most of the Wheeler-Kiladis-Takayabu spectrum of convectively coupled waves (Takayabu, 1994; Wheeler and Kiladis, 1999) in terms of linear wave theory (KM06a, KM08a, Han and Khouider (2010)) and nonlinear organization of large-scale envelopes mimicking cross-scale interactions of the Madden-Julian oscillation (MJO) and convectively coupled waves (KM07; KM08b; Majda et al. (2007)), in the idealized context of a simple twobaroclinic modes model employed here. Both the deterministic and stochastic multicloud models dramatically improve the representation of the coherent and intermittent nature of organized convection. This has been shown in an idealized two baroclinic mode framework coupled to a coarse resolution GCM for both the MJO and monsoon intraseasonal oscillations (Khouider et al., 2011; Deng et al., 2014; Ajayamohan et al., 2013, 2014; Deng et al., 2014). 
Here, a version of the stochastic multicloud parameterization (FMK13) is augmented with cloud radiation feedback and ocean coupling mechanism. It is natural to use the stochastic, rather than deterministic, multicloud parameterization for this purpose; this is because cloud fractions play such an important role in cloud-radiation feedback, and cloud fractions were introduced into the multicloud model as part of the stochastic parameterization. As in FMK13, the parameterization is coupled to a simplified model of the primitive equations; the vertical resolution is reduced to the first two baroclinic modes. Such a setup is intermediate between overly simple one baroclinic mode models (Tian and Ramanathan, 2003; Sobel et al., 2004) and more complex GCM and CRM simulations (Zurovac--Jevtik et al., 2005; Moncrieff and Klinker, 1997). The impact of radiative convective feedback of each of the three cloud types is parameterized through a product of the cloud fraction and two parameters, which represent the idealized projection of the cloud radiative feedback effect onto the two baroclinic modes of the system. The radiative feedback is shown to increase the strength and variability of the Walker circulation. The atmosphere-ocean coupling increases the variability of convection by introducing low frequency envelopes of synoptic and mesoscale convective systems. Single column simulations are used here to isolate and elucidate the effects of these modifications. Atmosphereocean coupling and cloud radiation feedback have a subtle but significant effect in spatially extended simulations. Spectral analysis highlights the effects of the modifications introduced here and their interactions with intrinsic variability of the system.

The remainder of the paper is organized as follows. A self-contained review of the stochastic multicloud parameterization is presented in Section 2. The section also introduces cloud radiation feedback and atmosphere ocean coupling mechanisms. In Section 3, single column simulations are used to illustrate the effects of the two modifications listed above. In Section 4, the modified parameterization is used to study flows above the equator in a series of idealized Walker cell simulations. Some discussion and concluding remarks are given in Section 5. 


\section{Multicloud model, cloud radiation feedback and atmosphere-ocean coupling}

We start with a brief review of the dynamical core equations used for the stochastic multicloud parameterization in Section 2.1. A more thorough and detailed discussion of the model equations is found in (KM06). Nevertheless, a comprehensive list of the model constants and parameters is given in Table 1 for the sake of completeness. Section 2.2 reviews the stochastic multicloud parameterization, while Sections 2.3 and 2.4 introduce cloud radiative feedback and ocean coupling, respectively.

\subsection{Dynamical core}

The stochastic multicloud parameterization assumes three heating profiles associated with the main cloud types that characterize organized tropical convective systems (Johnson et al., 1999): cumulus congestus clouds that heat the lower troposphere and cool the upper troposphere, through radiation and detrainment, deep convective towers that heat the whole tropospheric depth, and the associated lagging-stratiform anvils heat the upper troposphere and cool the lower troposphere, due to evaporation of stratiform rain. Accordingly, the dynamical core used in this paper consists of two coupled and forced shallow water systems. Without the meridional dependency, the equations are given by

$$
\begin{gathered}
\partial_{t} u_{j}-\partial_{x} \theta_{j}=C_{d} u_{0} u_{j}-\frac{1}{\tau_{R}} u_{j}, \quad j=1,2 \\
\partial_{t} \theta_{1}-\partial_{x} u_{1}=H_{d}+\xi_{s} H_{s}+\xi_{c} H_{c}+S_{1}, \\
\partial_{t} \theta_{2}-\frac{1}{4} \partial_{x} u_{2}=H_{c}-H_{s}+S_{2} .
\end{gathered}
$$

Here $H_{d}, H_{s}$ and $H_{c}$ are the heating rates for deep, stratiform and cumulus congestus clouds obtained by either the deterministic or the stochastic parameterization. These heating rates are combined to form the bulk precipitation $P=H_{d}+\xi_{s} H_{s}+\xi_{c} H_{c}$. The coefficients $\xi_{c}$ and $\xi_{s}$ denote contribution of congestus and stratiform rain to the bulk precipitation. The parameters $C_{d}$ and $u_{0}$ are respectively the momentum drag coefficient and the strength of turbulent 
fluctuations in the boundary layer. The last terms of the first and second baroclinic heating mode equations, $S_{j}, j=1,2$, represent radiative source terms, including cloud-radiation feedback, and will be discussed in Section 2.3.

The multicloud models additionally carry an equation for the vertically integrated tropospheric moisture content, $q$, and an equation for the boundary layer equivalent potential temperature, $\theta_{e b}$.

$$
\begin{gathered}
\partial_{t} q+\partial_{x}\left[\left(u_{1}+\tilde{\alpha} u_{2}\right) q+\left(u_{1}+\tilde{\lambda} u_{2}\right) \tilde{Q}\right]=-\frac{2 \sqrt{2}}{\pi} P+D / H_{T} \\
\partial_{t} \theta_{e b}=\frac{1}{h_{b}}(E-D) .
\end{gathered}
$$

The sea surface saturation equivalent potential temperature, $\theta_{e b}^{*}\left(T_{s}\right)$, is a function of the bulk ocean layer temperature discussed in Section 2.4 . For simulations without AOC, $\theta_{e b}^{*}\left(T_{s}\right)$ is set to constant, so that $\bar{\theta}_{e b}^{*}-\bar{\theta}_{e b}=10 K$. Here and throughout the paper, $\bar{X}$ denotes the radiative-convective equilibrium (RCE) value of the variable $X$. Notice that the simple treatment (5) of the boundary layer does not explicitly include the mechanism of Lindzen and Nigam (1987). In the $(x, t)$ simulations where SST is not homogenous, the sea surface evaporation $E$ takes the form

$$
\frac{E}{h_{b}}=\tau_{e}^{-1}\left(\theta_{e b}^{*}\left(T_{s}\right)+\theta_{e b \triangle}^{*}(x)-\theta_{e b}\right) .
$$

The sea surface saturation equivalent potential temperature takes the form

$$
\theta_{e b \triangle}^{*}(x)=5 \cos \left(\frac{4 \pi x}{40000}\right)+10 K,
$$

within an interval of 20,000 $\mathrm{km}$ of the $40,000 \mathrm{~km}$ domain and $\theta_{e b}^{*}=5 \mathrm{~K}$ everywhere else as in Khouider and Majda (2007) and KM08a. This setup mimics the Indian Ocean-Western Pacific warm pool.

\subsection{Stochastic multicloud parameterization}

The stochastic multicloud parameterization is designed to capture the dynamical interactions between the three cloud types that characterize organized tropical convection and the environment. In the stochastic multicloud model these interactions are represented through a coarse grained lattice model (KBM10). 


\begin{tabular}{|c|c|c|}
\hline Parameter & Value & Description \\
\hline$h_{b} / H_{m} / H_{T}$ & $500 \mathrm{~m} / 5 \mathrm{~km} / 16 \mathrm{~km}$ & depth of ABL/ mid-troposphere/ free troposphere \\
\hline$\xi_{s} / \xi_{c}$ & $0.4 / 0$ & Stratiform/Congestus contribution to first baroclinic mode \\
\hline$\tilde{Q}$ & 0.9 & Background moisture stratification \\
\hline$\tilde{\lambda} / \tilde{\alpha}$ & $0.8 / 0.1$ & Coefficient of $u_{2}$ in linear / nonlinear moisture convergence \\
\hline$m_{0}$ & Determined at RCE & Large-scale background downdraft velocity scale \\
\hline$\mu$ & 0.25 & Contribution of convective downdrafts to $D$ \\
\hline$\alpha_{s} / \alpha_{c}$ & $0.25 / 0.1$ & Stratiform/Congestus adjustment coefficient \\
\hline$\tau_{R} / \tau_{D}$ & 75 days / 50 days & Rayleigh drag / Newtonian cooling time scale \\
\hline$\tau_{s} / \tau_{c}$ & 3 hours / 2 hour & Stratiform / Congestus adjustment time scale \\
\hline$\tau_{\text {conv }}$ & 2 hours & Convective time scale \\
\hline$\tau_{e}$ & Determined by RCE & Surface evaporation time scale \\
\hline $\bar{Q}$ & Determined at $\mathrm{RCE}$ & Bulk convective heating at $\mathrm{RCE}$ \\
\hline $\bar{\theta}_{e b}-\bar{\theta}_{e m}$ & $11 \mathrm{~K}$ & Mean (RCE) Dryness of the atmosphere \\
\hline$a_{1} / a_{2}$ & $0.45 / 0.55$ & Relative contribution of $\theta_{e b} / \mathrm{q}$ to deep convection \\
\hline$a_{0} / a_{0}^{\prime}$ & $2 / 1.5$ & Dry convective buoyancy frequency in deep/congestus eqns. \\
\hline$\gamma_{2} / \gamma_{2}^{\prime}$ & $0.1 / 2$ & Relative contribution of $\theta_{2}$ to deep / congestus heating \\
\hline$\alpha_{2}$ & 0.1 & Relative contribution of $\theta_{2}$ to $\theta_{e m}$ \\
\hline$C_{d}$ & 0.001 & Surface drag coefficient \\
\hline$u_{0}$ & $2 \mathrm{~m} / \mathrm{s}$ & Strength of turbulent fluctuations \\
\hline$C A P E_{0}$ & $400 \mathrm{~J} / \mathrm{Kg}$ & Reference values of CAPE \\
\hline$T_{0}$ & $12 \mathrm{~K}$ & Reference values of dryness \\
\hline $\bar{\alpha}$ & $\approx 15 \mathrm{~K}$ & Unit scale of temperature \\
\hline$R_{\text {univ }}$ & $\approx 8.31436 \mathrm{~J} / \mathrm{mole} \mathrm{K}$ & Gas constant (universal) \\
\hline$R_{d}$ & $\approx 287.04 \mathrm{~J} / \mathrm{kg} \mathrm{K}$ & Gas constant (dry air) \\
\hline$R_{v}$ & $\approx 1461.50 \mathrm{~J} / \mathrm{kg} \mathrm{K}$ & Gas constant (water vapor) \\
\hline$T_{r e f}$ & $\approx 301 \mathrm{~K}$ & Constant reference temperature \\
\hline$L_{v}$ & $\approx 2.43510^{6} \mathrm{~J} / \mathrm{kg}$ & Latent heat of vaporization \\
\hline$c_{p}$ & $\approx 1005 \mathrm{~J} / \mathrm{K} \mathrm{kg}$ & Heat capacity of dry air \\
\hline
\end{tabular}

Table 1: Constants and parameters for multicloud parameterizations. 


\begin{tabular}{ll}
\hline Description & Expression \\
\hline Midlevel $\theta_{e m}$ & $\theta_{e m}=q+\frac{2 \sqrt{2}}{\pi}\left(\theta_{1}+\alpha_{2} \theta_{2}\right)$ \\
Precipitation & $P=H_{d}+\xi_{s} H_{s}+\xi_{c} H_{c}$ \\
Downdrafts & $D=m_{0}\left(1+\mu\left(H_{s}-H_{c}\right) / Q_{R 01}\right)^{+}\left(\theta_{e b}-\theta_{e m}\right)$ \\
Radiation & $\operatorname{Rad}_{1}=Q_{R 01}-\frac{\theta_{1}}{\tau_{D}}, \quad$ and $\quad \operatorname{Rad}_{2}=Q_{R 02}-\frac{\theta_{2}}{\tau_{D}}$ \\
CAPE & $C A P E=\overline{C A P E}+R\left(\theta_{e b}-\gamma\left(\theta_{1}+\gamma_{2} \theta_{2}\right)\right)$ \\
Lower level CAPE & $C A P E_{l}=\overline{C A P E}+R\left(\theta_{e b}-\gamma\left(\theta_{1}+\gamma_{2}^{\prime} \theta_{2}\right)\right)$ \\
Deep heating & $H_{d}=\left[\sigma_{d} \bar{Q}+\frac{\sigma_{d}}{\bar{\sigma}_{d} \tau_{c}^{0}}\left(a_{1} \theta_{e b}+a_{2} q-a_{0}\left(\theta_{1}+\gamma_{2} \theta_{2}\right)\right)\right]^{+}$ \\
Congestus heating & $H_{c}=\sigma_{c} \frac{\alpha_{c} \bar{\alpha}}{H_{m}} \sqrt{C A P E_{l}^{+}}$ \\
\hline
\end{tabular}

Table 2: Summary of important diagnostic quantities in multicloud model. For more details, the reader is referred to FMK13.

To mimic the behavior of convective variability within a typical GCM grid box, a rectangular $n \times n$ lattice is considered. Each element of the lattice is occupied by a congestus, deep or a stratiform cloud or is a clear sky site. It is represented by an order parameter that takes accordingly the values $0,1,2$ or 3 . A continuous time stochastic process is then defined by allowing the transitions, for individual cloud sites, from one state to another according to intuitive probability transition rates, which depend on the resolved ("large scale") variables. These large scale variables are the convective available potential energy integrated over the whole troposphere (CAPE), low level CAPE (see Table 2) and the dryness of the mid troposphere, which are themselves functions of the multicloud model variables such as $\theta_{e b}, \theta_{1}, \theta_{2}, q$, etc. For example, the dryness of the mid troposphere is a function of the difference between the atmospheric boundary layer (ABL) temperature $\theta_{e b}$ and the middle tropospheric potential temperature $\theta_{e m}$. The inclusion of the dryness of the middle troposphere accounts for mixing of the convective parcels with dry environmental air is conceptually similar to the 
Table 3: Transition rates and time scales in the stochastic multicloud model simulations. Here

$\Gamma(x)=1-\exp (-x)$ for $x>0$ and zero otherwise, $C_{l}=C A P E_{l} / C A P E_{0}, C=C A P E / C A P E_{0}$ and $D=\left(\theta_{e m}-\theta_{e b}\right) / T_{0}$. For more details, the reader is referred to FMK13.

\begin{tabular}{|c|c|c|}
\hline Description & Transition Rate & Time scale (h) \\
\hline Formation of congestus & $R_{01}=\frac{1}{\tau_{01}} \Gamma\left(C_{l}\right) \Gamma(D)$ & $\tau_{01}=1$ \\
\hline Decay of congestus & $R_{10}=\frac{1}{\tau_{10}} \Gamma(D)$ & $\tau_{10}=1$ \\
\hline Conversion of congestus to deep & $R_{12}=\frac{1}{\tau_{12}} \Gamma(C)(1-\Gamma(D))$ & $\tau_{12}=1$ \\
\hline Formation of deep & $R_{02}=\frac{1}{\tau_{02}} \Gamma(C)(1-\Gamma(D))$ & $\tau_{02}=3$ \\
\hline Conversion of deep to stratiform & $R_{23}=\frac{1}{\tau_{23}}$ & $\tau_{23}=3$ \\
\hline Decay of deep & $R_{20}=\frac{1}{\tau_{20}}(1-\Gamma(C))$ & $\tau_{20}=3$ \\
\hline Decay of stratiform & $R_{30}=\frac{1}{\tau_{30}}$ & $\tau_{30}=5$ \\
\hline
\end{tabular}


switch $\Lambda$ in the deterministic multicloud model(KM06a, KM06b,KM07, KM08a, KM08b).

The probability rates are constrained by a set of intuitive rules which are based on observations of cloud dynamics in the tropics (e.g. Johnson et al. 175 (1999); Mapes (2000), KM06a, and references therein). Following KBM10, a clear site turns into a congestus site with high probability if low level CAPE is positive and the middle troposphere is dry. A congestus or clear sky site turns into a deep convective site with high probability if CAPE is positive and the middle troposphere is moist. A deep convective site turns into a stratiform site with high probability. Finally, all three cloud types decay naturally to clear sky at some fixed rate. All other transitions are assumed to have negligible probability. These rules are formalized in Table 3 . Notice that the assumption that the transition rates depend on the large scale variables accounts for the feedback of the large scales on the stochastic model, while ignoring the interactions between the lattice sites all together implies that the stochastic processes associated with the different sites are identical (independent and identically distributed). The latter simplification makes it easy to derive the stochastic dynamics for the GCM grid box cloud coverage alone, which can be evolved without the detailed knowledge of the micro-state configuration, by using a coarse-graining technique 190 (Katsoulakis et al., 2003,b) that yields in this case a system of three dimensional birth-death stochastic process for the congestus, deep and stratiform cloud fractions $\sigma_{c}, \sigma_{d}$ and $\sigma_{s}$ respectively (KBM10). The cloud fractions impact the large scale dynamics through algebraic congestus and deep heating closures, listed in Table 2, and dynamical closure for stratiform heating below,

$$
\partial_{t} H_{s}=\frac{1}{\tau_{s}}\left(\alpha_{s} \sigma_{s} H_{d} / \bar{\sigma}_{d}-H_{s}\right)
$$

More detailed description of the stochastic multicloud model can be found in FMK13. 


\subsection{Cloud Radiation Feedback}

Clouds plays a key role in the understanding weather and climate. The stochastic multicloud model outlined in the previous section does a remarkable

convective activity. We define cloud radiative forcing as the difference between the radiative heatings for cloud and clear skies. Positive cloud forcing implies 
the presence of the cloud warms the atmosphere relative to a clear sky heating profile. In order to infer the effects of cloud radiative forcing, we consider some observational analyses (Cox and Griffith, 1979a,b).

As shown in the observational analysis of Cox and Griffith (1979a) and in CRM studies (Peters and Bretherton, 2005), within the deep cloud, the longwave and shortwave heating are both essentially zero. The long-wave cooling at cloud top apparently overwhelms the shortwave warming there, and the result is slight cooling in the first baroclinic mode and more cooling in the upper troposphere than the lower troposphere. For stratiform and deep convective clouds, longwave cloud top cooling is roughly balanced by shortwave cloud top warming during daytime. As a result deep convective clouds warm the lower troposphere relative to the upper troposphere due to longwave cloud base warming in the lower troposphere (Cox and Griffith, 1979a,b). This results in the positive projection onto both first and second baroclinic modes to create a heating profile biased towards lower troposphere. By the same reasoning (Cox and Griffith, 1979a,b; Peters and Bretherton, 2005), stratiform clouds, which follow the deep convection, warm the upper troposphere relative to the lower troposphere due to longwave cloud base warming in the upper troposphere. This corresponds to the choice of heating profile slightly biased towards upper level heating through negative second baroclinic mode contribution. The evidence for effects of congestus cloud forcing is less clear (Cox and Griffith, 1979b). The congests clouds do not extend in the upper troposphere, and therefore the forcing here is zero. 250 The observational evidence (Schumacher et al., 2004) points to wards cooling of the lower troposphere relative to the upper troposphere by congestus clouds due to longwave cloud top cooling. This corresponds to cooling in of both first and second baroclinic modes by congestus radiative feedback.

The cloud radiative forcing inferred from the observations above is projected 255 on two the baroclinic modes of variability in the model. Formally, we propose the following parameterization for radiative flux terms $S_{i}, i=1,2$ in equations 1 :

$$
S_{i}=-\theta_{i} / \tau_{D}+\sigma_{c s} R_{i}^{\text {clear }}+\left(\sigma_{c} R_{i}^{c}+\sigma_{d} R_{i}^{d}+\sigma_{s} R_{i}^{s}\right) \alpha, i=1,2
$$


Here, $\sigma_{c s}=1-\sigma_{c}-\sigma_{d}-\sigma_{s}$, is clear sky fraction. We also introduce tuning parameter, $\alpha$. Setting $\sigma_{c s}=1$ and $\alpha=0$ reduces radiative flux to a simpler cipitation efficiency parameter), is commonly a part of the radiation scheme in both simple one baroclinic mode model (Sobel et al., 2004) and CRM studies 
(Bony and Emanuel, 2005; Zurovac-Jevtik et al., 2005).
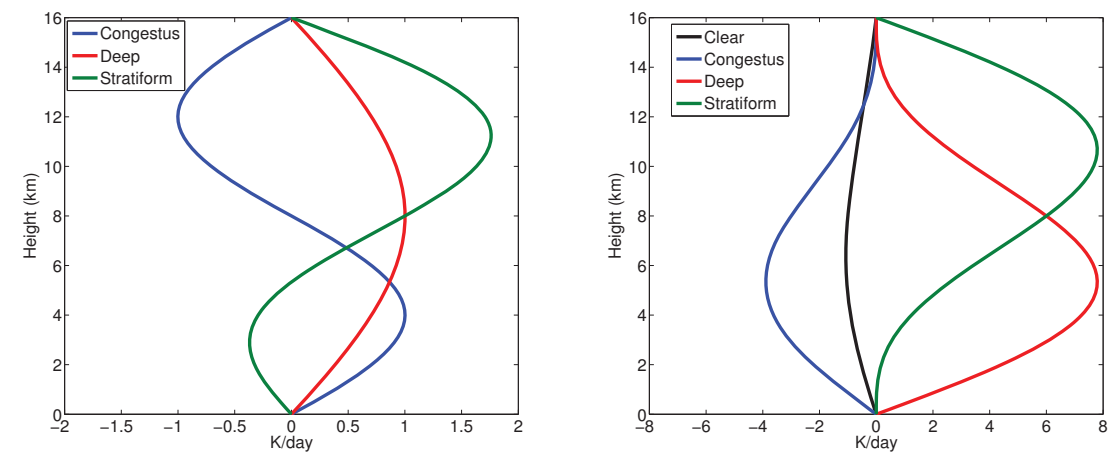

Figure 1: Left: Idealized convective heating of the three cloud types. Since there is no congestus precipitation in this parameter regime $\left(\xi_{c}=0\right)$, congestus heating projects purely on second baroclinic mode. Deep convection projects only on the first baroclinic mode. Stratiform rain projects onto the first baroclinic (through $\xi_{s}=0.5$ ) in addition to warming the upper troposphere, thus resulting in profile skewed towards the upper troposphere heating. Right: schematics of cloud radiative forcing profile computed from each cloud type and clear sky. The schematic is computed by imposing values in Table 4 on the two baroclinic modes and (for ease of comparison) assuming that each cloud completely covers the sky. The clear sky cooling rate is of profile typically used in the multicloud models.

\subsection{Slab mixed-layer ocean}

We use a simple model for an interactive bulk mixed-layer ocean. The character of our mixed-layer ocean will be subsumed into a single variable, $T_{s}$, the sea surface mixed layer temperature. The evolution of $T_{s}$ will be given by

$$
c_{0} \frac{d T_{s}}{d t}=S-\frac{c_{0}}{\tau_{e o}}\left(\theta_{e b}^{\star}\left(T_{s}\right)-\theta_{e b}\right)
$$

where $c_{o}$ is the heat capacity for the mixed layer ocean. The radiation flux, $S=S^{\text {rad }}+S^{\text {out }}$, includes radiative effects, $S^{\text {rad }}$ and an imposed ocean heat transport, $S^{\text {out }}$. The value of the ocean layer evaporation constant, $\tau_{e o}$ is determined by RCE conditions, and the surface temperature variable $T_{s}$ is an anomaly from equilibrium conditions (i.e., it takes the value of zero in RCE). 
Table 4: Parameters and constants used for the cloud radiative feedback and ocean coupling. Note that $R_{2}^{\text {clear }} \approx 0.3 \mathrm{~K} /$ Day is determined by RCE and is comparable to the value used in the previous multicloud model studies.

\begin{tabular}{|l|l|l|}
\hline \hline Parameter & CRF rate coefficient associated with & value \\
\hline$R_{1}^{c}$ & $\begin{array}{l}\text { Congestus clouds for 1st baroclinic } \\
\text { mode }\end{array}$ & $-3 \mathrm{~K} /$ day \\
\hline$R_{1}^{d}$ & Deep clouds for 1st baroclinic mode & $6 \mathrm{~K} /$ day \\
\hline$R_{1}^{s}$ & $\begin{array}{l}\text { Stratiform clouds for } 1 \text { st baroclinic } \\
\text { mode }\end{array}$ & $6 \mathrm{~K} /$ day \\
\hline$R_{1}^{\text {clear }}$ & Clear sky for 1 st baroclinic mode & $-1 \mathrm{~K} /$ day \\
\hline$R_{2}^{c}$ & $\begin{array}{l}\text { Congestus clouds for } 2 \text { nd baroclinic } \\
\text { mode }\end{array}$ & $-1.5 \mathrm{~K} /$ day \\
\hline$R_{2}^{d}$ & Deep clouds for 2nd baroclinic mode & $3 \mathrm{~K} /$ day \\
\hline$R_{2}^{s}$ & $\begin{array}{l}\text { Stratiform clouds for 2nd baroclinic } \\
\text { mode }\end{array}$ & $-3 \mathrm{~K} /$ day \\
\hline$R_{2}^{\text {clear }}$ & Clear sky for 2nd baroclinic mode & $-0.2 \mathrm{~K} /$ day \\
\hline
\end{tabular}


The sensible heat flux will be ignored, since its contribution to the energy budget is small compared to the shortwave radiation and latent heat fluxes (Peters and Bretherton, 2005; Sobel et al., 2004; Kikuchi and Wang, 2008; Frenkel et al., 2010).

In their model for Walker circulation, Sobel et al. (2004) used a similar mixed-layer ocean model. The authors used zonally varying term for the combined effect of ocean heat transport and clear sky shortwave forcing. Also, a similar model with zonally varying ocean heat transport has been used in Peters and Bretherton (2005). Here, we omit explicit representation of the ocean heat transport and instead use equation 7 to account for zonal SST variation implicitly, through $E$. This facilitates a direct comparison to previous multicloud 310 studies.

As shown in Peters and Bretherton (2005); Sobel et al. (2004), the long-wave cooling of the surface is nearly cancelled by back radiation from the atmosphere. Due to the smallness of longwave cloud forcing we will not consider long-wave radiation for cloud forcing at the surface, but will include long-wave effects in the constant clear sky flux. For the radiative flux $S^{\text {rad }}$ at the surface, we will have a clear sky component $S^{\text {clear }}$ and a cloud forcing component $S^{c f}$ :

$$
S^{\text {rad }}=S^{\text {clear }}+S^{c f}
$$

For clear sky, the net shortwave flux averaged over one day is roughly $S^{\text {clear }}=300 \mathrm{~W} / \mathrm{m}^{2}$, as shown in Sobel et al. (2004); and the net long-wave flux is roughly $S_{l w}^{c l e a r}=-50 \mathrm{~W} / \mathrm{m}^{2}$, as shown in Peters and Bretherton (2005). Therefore, we will choose the daily-averaged clear sky flux to be

$$
S^{\text {clear }}=S_{l w}^{\text {clear }}+S_{s w}^{c l e a r}
$$

For the radiative cloud forcing, we use

$$
S^{c f}=-S_{s w}^{c l e a r}\left(\sigma_{c}+\sigma_{d}+0.5 \sigma_{s}\right)
$$

In the equation above, we make an assumption that stratiform clouds, due to their relative thinness, block half as much short wave radiation compared to the 
thicker congestus and deep clouds. However, a large area fraction of stratiform

clouds makes them extremely important for radiative feedback effects.

We can further rewrite equation 10 in an explicit form.

$$
\frac{d T_{s}}{d t}=\frac{S^{c l e a r}\left(1-A^{c}\right)-S^{s w} A^{c}}{c_{0}}-\frac{1}{\tau_{e o}}\left(\theta_{e b}^{\star}\left(T_{s}\right)-\theta_{e b}\right)
$$

Here we use the sum of cloud area fractions to determine fraction cloud coverage of the sky $A^{c}=\left(\sigma_{c}+\sigma_{d}+0.5 \sigma_{s}\right) \alpha_{o}$, along with a tuning parameter $\alpha_{o}$. This tuning parameter is similar to the tuning parameter $\alpha$ used for radiative feedback coupling in equation 9. The corresponding radiative factor for convective clouds is given by $S^{s w} \approx 200 \mathrm{~W} / \mathrm{m}^{2}$, as estimated above. The mixed layer heat capacity $c_{0}$ is given by

$$
c_{0}=c_{R, 0} \rho_{0} h_{m l}
$$

This calculation is based on (heat capacity) $c_{R, 0}=4000 \mathrm{~J} / \mathrm{kg} \mathrm{K}$ and density $\rho$. When depth of mixed layer, $h_{m l}$, is set to 20 meters, $c_{0} \approx 8 \times 10^{7} \mathrm{JK}^{-1} \mathrm{~m}^{-2}$. This mixed layer depth corresponds to approximately 40 day time scale for mixed ocean layer. In select simulations presented here, we will vary mix layer depth, by setting $h_{m l}=10,20$ and 40 meters, which results in AOC time scale of 20,40 and 80 days, respectively, for mixed layer. The ocean layer $T_{s}$ is coupled to the atmospheric boundary layer $\left(\theta_{e b}\right)$ through atmospheric boundary layer saturation equivalent potential temperature. A simple closure, $\theta_{e b}^{\star}\left(T_{s}\right)=5 T_{s}$, is derived from the Clausius-Clapeyron relation as shown in Appendix A. In simulations without ocean, saturation equivalent potential temperature, $\theta_{e b}^{\star}$, is independent of $T_{s}$.

\section{Single column simulations}

In this section, the effects of the new mechanisms of cloud-radiation feedback and atmosphere-ocean coupling, are studied in the context of single column simulations and compared to the FMK13 results. The single column equations are obtained by disregarding spatial dependence components and the zonal wind. 
As in KBM10, we employ a third order Adams-Bashforth method to integrate

in time by means of Gillespie's exact algorithm (Gillespie, 1975, 1977). All simulations in this section are run for 2000 days, while a 10 or 100 days interval of the solution is shown.

To facilitate the comparison, we first review the basic results of FMK13. the time synchronization of the oscillations of the stochastic and deterministic variables which leads to time series with frequent precipitation peaks of 10 $\mathrm{K} /$ Day and more intermittent large precipitation events on the order of 20 K/Day. Each convective event is initialized by a build up of low level CAPE. combined with the build up of CAPE, produces deep convective events which are in turn followed by stratiform clouds. The relationship between small and large precipitation events is reminiscent of a progressive deepening of convection on multiple scales (Mapes et al. 2006). By design, the congestus clouds are followed by deep convective and trailing stratiform clouds. The transitions rates are associated with moisture and dryness. In particular, we see a high correlation between positive, $\theta_{e b}$ and moisture anomalies and deep convective activities. In this simulation, the ocean mixed layer temperature equation is slaved to the atmospheric variables and does not feedback into the model dynamics (which are driven under the assumption that $T_{s}$ is actually fixed at its equilibrium value).

Figures 3 shows the time series of simulations with cloud radiation feedback and atmosphere ocean layer and with radiative convective feedback. We choose small value of CRF strength $(\alpha=0.1)$ and 40 meter deep ocean layer. The top panel shows the contribution of the radiative feedback to the heating. The effects of radiative convective feedback are subtle. The feedback accounts for roughly one tenths of the heating in the column, which most significant contribution coming from large deep convective events and trailing stratiform anvils. On the other hand, the atmosphere ocean coupling produces pronounced en- 
be shown in the following figures), associated with the ocean temperature fluctuations (bottom panel). The clear sky conditions lead to increase in mixed ocean layer temperature, which leads to increase in ABL temperature anomalies, and creation of deep convection and stratiform anvils, which cools mixed

velope of convective activity with roughly 30 day period of oscillation (as will ocean layer, in turn, leading to smaller convective fraction and clear sky, closing the loop. Overall, the dynamics of the model becomes more irregular with introduction of ocean coupling and CRF. In fact the model has suppressed and enhanced convection periods associated with time scale related to the ocean depth. The direct link between the ocean layer depth and the intraseasonal and subannual oscillations will be discussed below in more detail. Briefly, the oceanic dynamics introduces a new time scale, $\tau_{e o}$, into the system, and this atmosphere-ocean coupling time scale is relatively slow and introduces low frequency oscillations. These oscillations resemble suppressed and active phases of MJO but on different time scales. We note that, just like in MJO, the suppressed phase of oscillation has intermittent deep convection and small average cloud fraction, while the active phase of oscillation consists of highly organized coherent convective episodes.

In Figure 4 we study the response of the model to variation in the strength of the CRF in presence of the 20 meter deep ocean layer coupling. For reference, panel A shows results from FMK13 (see Figure 3 in FMK13). The mixed ocean layer is added in simulations of panel B, we note that ocean coupling introduces weak low frequency envelope to the time series. It can seen that the period of oscillation is on order of 30 days. The oscillation does not seem to be associated with a deterministic instability since, in a linear stability analysis (not shown) of a deterministic version of the model, there is a stable RCE state. The addition of weak CRF coupling $(\alpha=0.1)$, in panel $\mathrm{C}$, enhances this effect but shortens the frequency of the oscillation. It also makes oscillations in the ocean mixed layer more chaotic. The strong CRF coupling ( $\alpha=0.4)$, of panel D, destroys the low frequency envelope. The values of $\alpha$ higher than approximately 0.4 produce unphysical large climatology. This is somewhat similar to, but not as extreme 
as, the findings of Sobel et al. (2004), where strong values of the convective feedback tuning parameter were seen to cause solutions to blow up.

In Figure 3, we experiment with the depth of the ocean mixed layer, while keeping the cloud radiation feedback constant ( at weak value of $\alpha=0.1$ ). It appears that the mixed ocean layer envelope modulation is prominent for all ocean depths considered. To quantify the changes in the behavior we study the Fourier spectrum of the precipitation in Figure 5. Firstly, we observe that ocean coupling introduces low frequency oscillation. It could further be established that the time scale of this oscillation is proportional to the depth of the layer. Secondly, we draw the conclusion that strong convective radiative feedback interferes with the low frequency introduced by the ocean mixed layer. The stronger values of radiative feedback lead to shift of the power in the spectrum from low frequency oscillations associated with ocean layer to higher frequency intrinsic variability. While the results are omitted for conciseness, in absence of ocean coupling, radiative feedback slightly shifts the power spectrum towards the modes with frequency slightly lower than the intrinsic variability of the system but shorter than frequencies associated with bulk ocean layer. The Fourier spectrum analysis confirms observations made from Figure 4.

It is believed that both CRF and AOC play an important role in low fre430 quency tropical variability, such as MJO and ENSO (Bony and Emanuel, 2005). However, it is hard to infer the exact effects of CRF and AOC on these multiscale phenomena from column simulations. In order to investigate the behavior of the model further we proceed to the spatially extended simulations. 

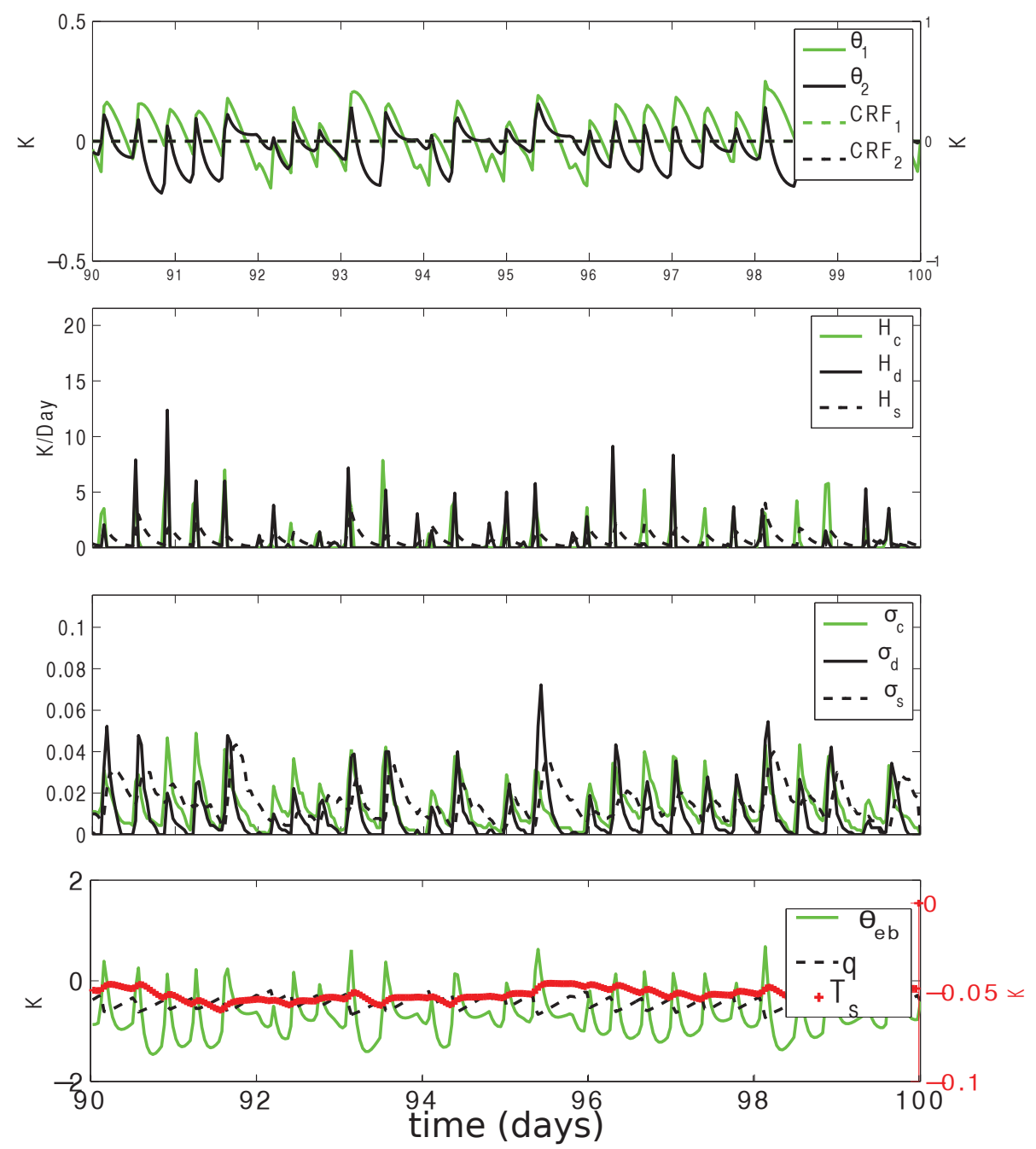

Figure 2: Time series of dynamic variables for FMK13. In this simulation, the ocean mixed layer temperature equation is a passive slave to the system and does not feedback into the model dynamics, and cloud-radiative feedback was not included and hence is plotted with amplitude 0. CRF1 and CRF2 refer to the cloud-radiation feedback portion of the radiative heating $S_{1}$ and $S_{2}$, respectively, of (9). 

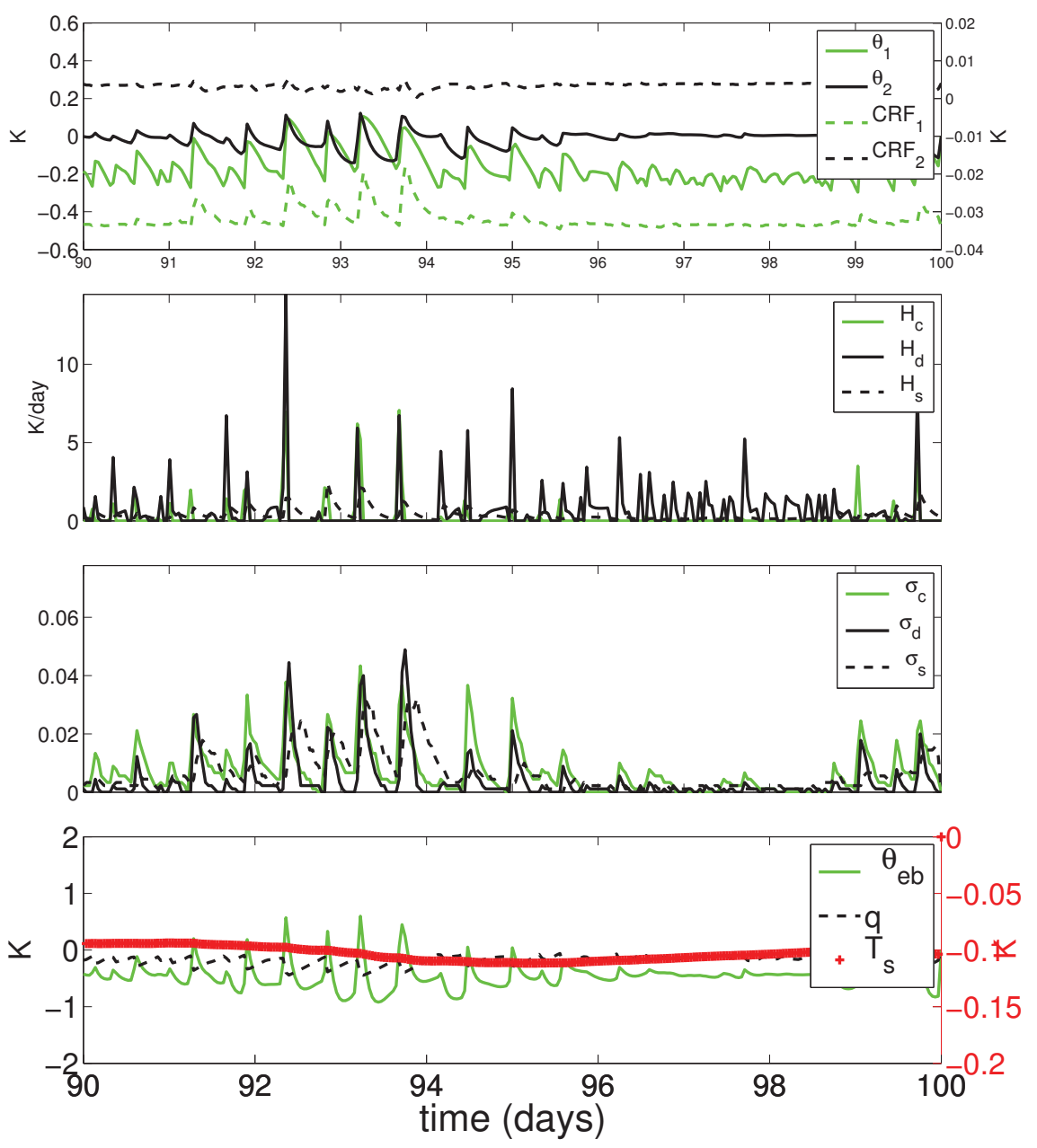

Figure 3: Same as Fig. 2, except with additions of cloud-radiation feedback with $\alpha=0.1$ and 40 meter deep ocean layer. The effects of cloud-radiation feedback (CRF1 and CRF2) are roughly one-tenth the magnitude of the convective heating $\left(H_{c}, H_{d}, H_{s}\right)$. Overall, the dynamics of the model is greatly improved by the introduction of the suppressed and enhanced convection periods associated with low frequency sea surface temperature oscillations. 

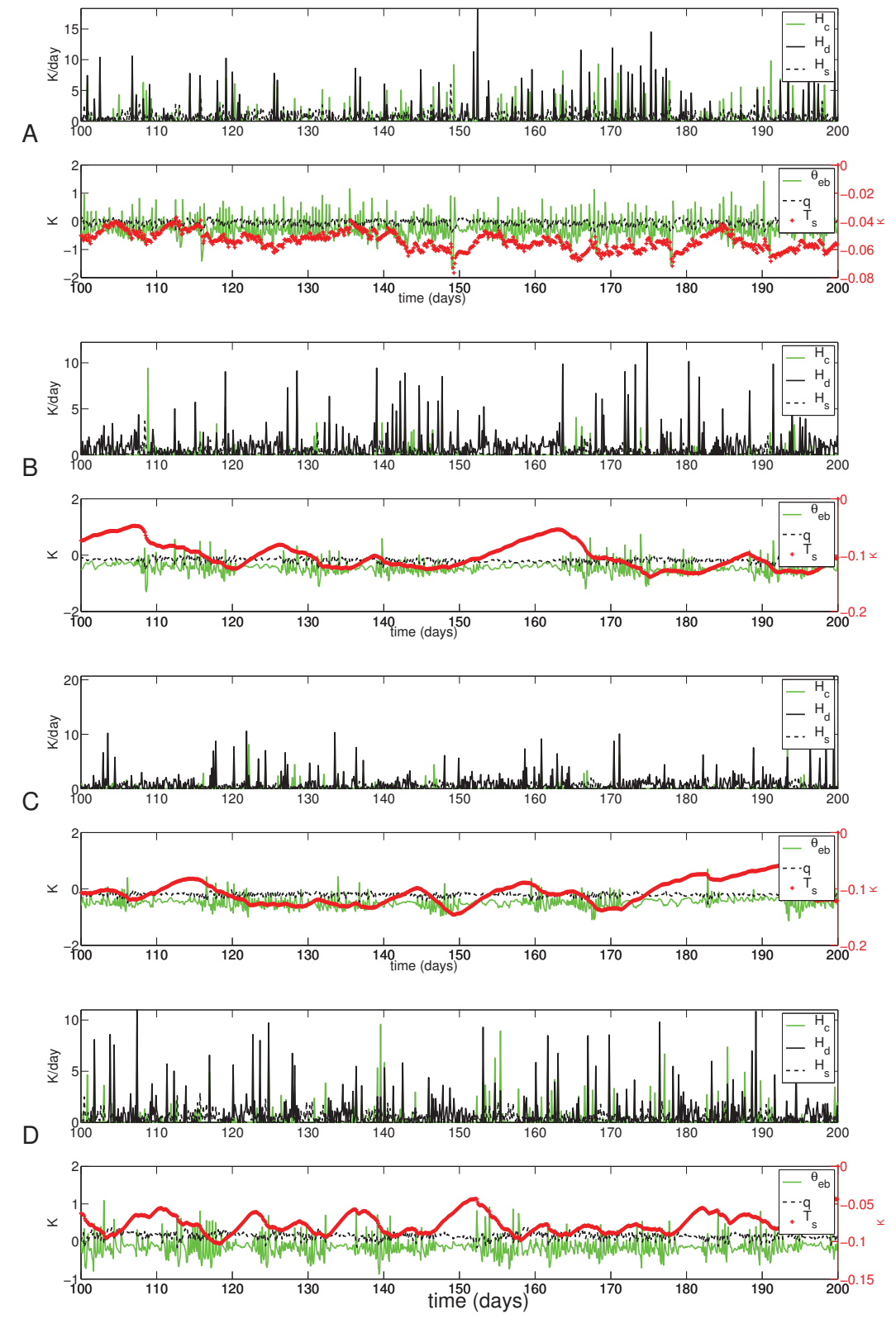

Figure 4: Precipitation and mixed ocean layer temperature for simulations (top to bottom) associated with: A) FMK13, B) simulations with atmosphere ocean coupling (20 meter) and without atmospheric cloud-radiative forcing $\alpha=0$, C) 20 m ocean with weak feedback $\alpha=0.1$ and D) $20 \mathrm{~m}$ ocean with strong radiative feedback $\alpha=0.4$. 


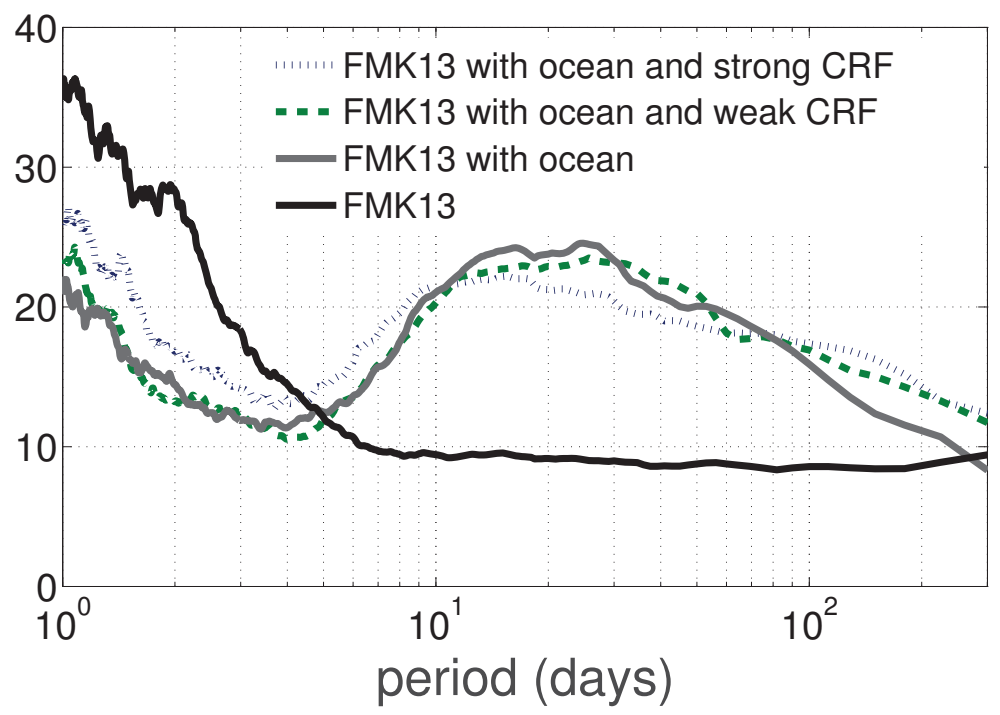

Figure 5: Power spectrum of precipitation time-series for column simulations with FMK13 (solid black), FMK13 with atmosphere-ocean coupling (20 meter) and without radiative feedback $\alpha=0$ (solid grey), FMK13 with atmosphere-ocean coupling (20 meter) and with weak radiative feedback $\alpha=0.1$ (green dash) and FMK13 with atmosphere-ocean coupling (20 meter) and with weak radiative feedback $\alpha=0.4$ (fine blue dash). This corresponds to four cases shown in Figure 4. 


\section{Spatially extended simulations with a warm pool}

435 stochastic multicloud model with non-uniform SST backgrounds, mimicking the Indian Ocean western Pacific warm pool (Section 2.4). Simulations with new mechanisms are considered and compared to FMK13, CRMs, and observations. We first consider separately variation of $\mathrm{CRF}$ and ocean layer depth before

contributes to the weaker mean Walker circulation. This is in sharp contrast 


\begin{tabular}{lllll}
\hline $\mathrm{CRF}$ & Ocean & $\mathrm{SST}$ & $(\mathrm{U}, \mathrm{W})$ & $\mathrm{std}\left(\mathrm{u}_{1}\right)$ \\
$\alpha$ & & & $(\mathrm{m} / \mathrm{s}, \mathrm{cm} / \mathrm{s})$, & $\mathrm{m} / \mathrm{s}$ \\
\hline 0 & - & $5 \mathrm{~K}$ & $(9.3,0.86)$ & 0.18 \\
0.1 & - & $5 \mathrm{~K}$ & $(9.7,0.97)$ & 0.19 \\
0.4 & - & $5 \mathrm{~K}$ & $(10.5,1.11)$ & 0.19 \\
0 & $10 \mathrm{~m}$ & $5 \mathrm{~K}$ & $(8.0,0.63)$ & 0.18 \\
0 & $20 \mathrm{~m}$ & $5 \mathrm{~K}$ & $(7.7,0.62)$ & 0.17 \\
0 & $40 \mathrm{~m}$ & $5 \mathrm{~K}$ & $(7.6,0.61)$ & 0.14 \\
0.4 & $40 \mathrm{~m}$ & $5 \mathrm{~K}$ & $(8.9,0.7)$ & 0.20 \\
0 & & & & \\
0.4 & $40 \mathrm{~m}$ & $2.5 \mathrm{~K}$ & $(4.3,0.46)$ & 0.20 \\
\hline
\end{tabular}

Table 5: Mean and variabilty of the Walker circulation in the spatially extended simulations. Each case uses different values of the cloud-radiation feedback parameter $\alpha$, ocean layer depth, and amplitude of the warm pool sea surface temperature. For the time-averaged Walker circulation, the statistics reported are the maxima of $U$ and $W$ and the standard deviation of $u_{1}$. 
with counterintuitive result of Sobel et al. (2004), where the authors propose recharge-discharge theory to account for an increased heating associated with AOC. On the other hand anomaly effects on wind evaporation feedback are completely absent in the present models. Also, since only the mixed ocean layer instead of ocean dynamics is included in this work, the cloud over the warm pool will reduce the shortwave radiation and cool the SST.

The Figure 7 shows deviations from zonal mean velocity field for all the regimes. We note that the simulations with the $\mathrm{CRF}$ produce the strongest intermittent bursts of convection. In particular the both weak and strong CRF ( $\alpha=0.1$ and 0.4 respective) simulations in panels B and $\mathrm{C}$ are more intermittent than the FMK13 simulation in panel A, in the sense that panel A displays a repetitive sequence of convectively coupled wave events, each of roughly the 475 same amplitude and repeating roughly every 15 days, whereas panels B and C display irregular periods of time between convectively coupled wave events and a mixture of periods of weak convectively coupled wave activity (such as times 870-890 days in panel B and times 700-740 days in panel C) and strong convectively coupled wave activity (such as times 830-850 days in panel B and times 860-890 days in panel C). These simulations also produce the strongest variability and mean, as shown in Table 5. The simulation with ocean (but without $\mathrm{CRF}$ ), shown in figure $\mathrm{D}$, shows and interesting low frequency variability on order of 100 days (i.e., periods of strong convectively coupled wave events at times $760-800,870-890$, and 920-940 days are separated by periods of relatively weak convectively coupled wave activity at times $700-760,800-870$, and 890 920 days). The remnants of this behavior can be seen when a weak CRF is also introduced in panel $\mathrm{E}$, but disappear with the stronger CRF of panel $\mathrm{F}$.

Figure 8 shows Fourier spectrum for velocity, atmospheric moisture, ABL and sea surface temperature. We note that variability for the base FMK13 case, is characterized by 15 day convectively coupled waves (while the moisture $q$ tends to have maximum power at a time scale of 7 or 8 days). These are associated with synoptic systems originating inside of the warm pool. A similar oscillation is observed by Slawinska et al. (2014b) in a CRM study. The shallower 
ocean layer shifts the variability towards (particularly in atmospheric and ABL moisture) lower frequency time scales. A similar effect is associated with lower SST gradient discussed in the following section. In all cases, the ocean layer decreases the strength of the variability and introduces low frequency envelope structures in atmospheric and ocean boundary layer fields. The Fourier analysis in Figure 8 shows a progressive shift towards the lower frequencies associated 500 with the increase in ocean depth. The same figures shows the effects of the CRF, which are mixed in nature. The radiative convective feedback allows for slightly larger amplitude variability but negatively impacts low frequency oscillations in the system.

Note that, we omit spatially extended homogeneous SST simulations, since it is hard to distinguish (visually) the difference between standard FMK13 simulations and the ones with low and moderate radiative convective feedback. We also performed simulations with wind induced surface heat exchange (WISHE) (Majda and Shefter, 2001). Likewise, these results are also omitted, since the addition of WISHE mechanism did not appear to a significant impact on the mean or the variability of the stochastic multicloud model simulations. 


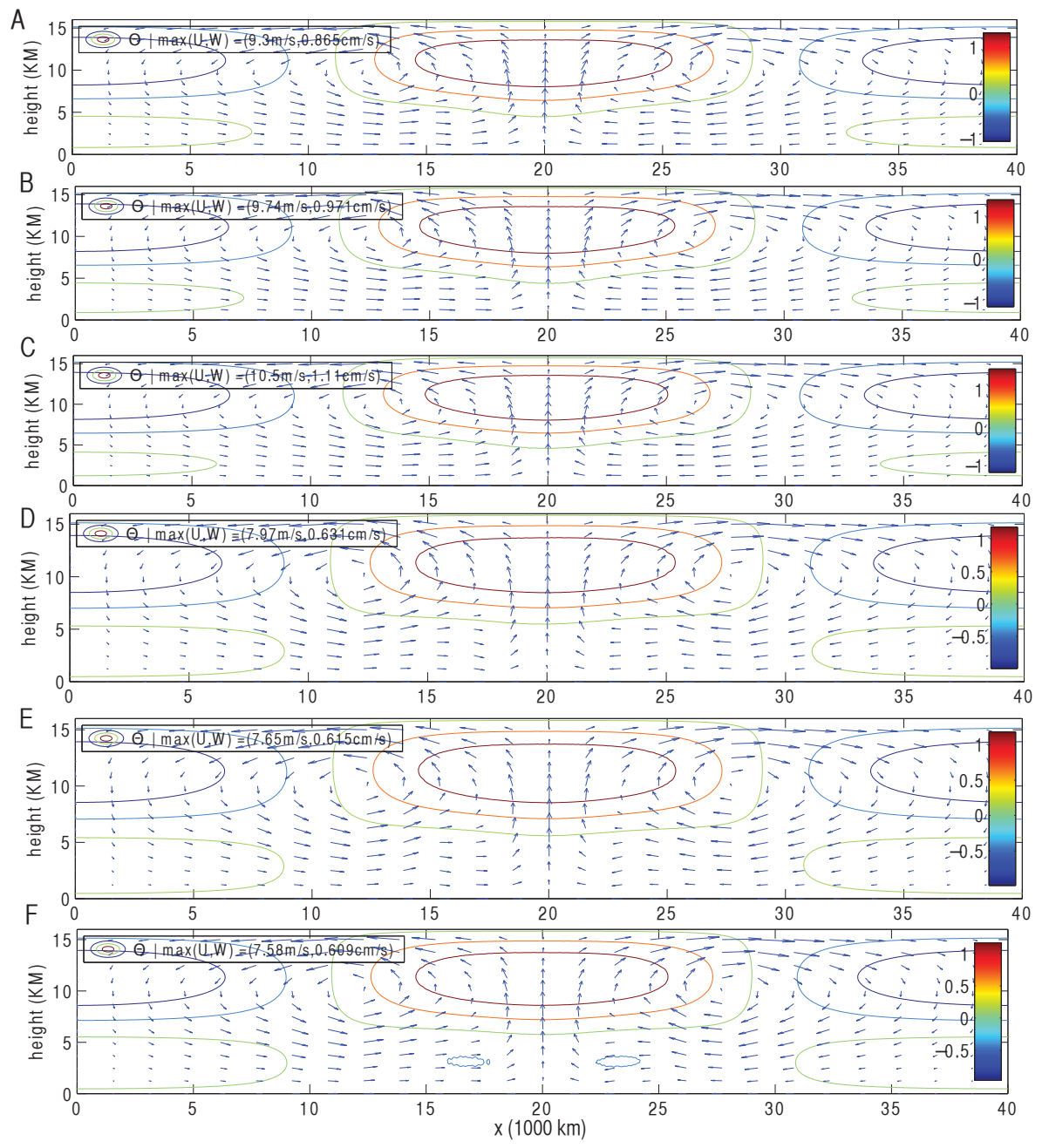

Figure 6: Mean zonal vertical structure associated with: A) FMK13 B) FMK13 with weak CRF $(\alpha=0.1)$ C) FMK13 with strong CRF $(\alpha=0.4)$, D) FMK13 with 10 meter ocean, E) FMK13 with 20 meter ocean, F) FMK13 with 40 meter ocean . 

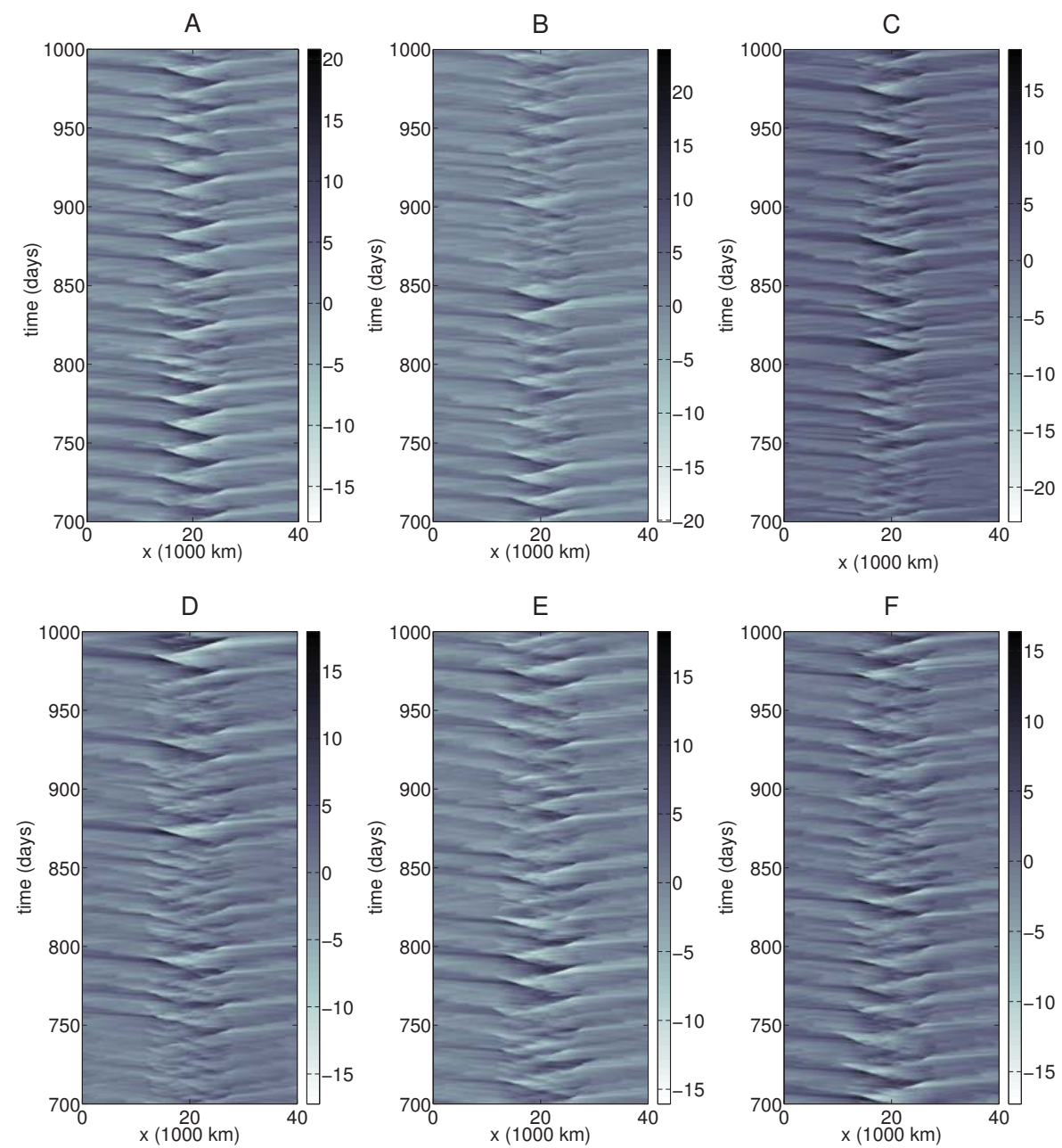

Figure 7: Contours of velocity anomalies $u_{1}(x, t)$ for: a) FMK13, b) FMK13 with weak CRF $(\alpha=0.1)$, c) FMK13 with strong CRF $(\alpha=0.4)$, d) FMK13 with 10 meter ocean , e) FMK13 with 20 meter, f) FMK13 with 40 meter ocean. Anomalies are computed as deviations from the time-averaged mean state. Note that slightly different color bars are used in different panels. 

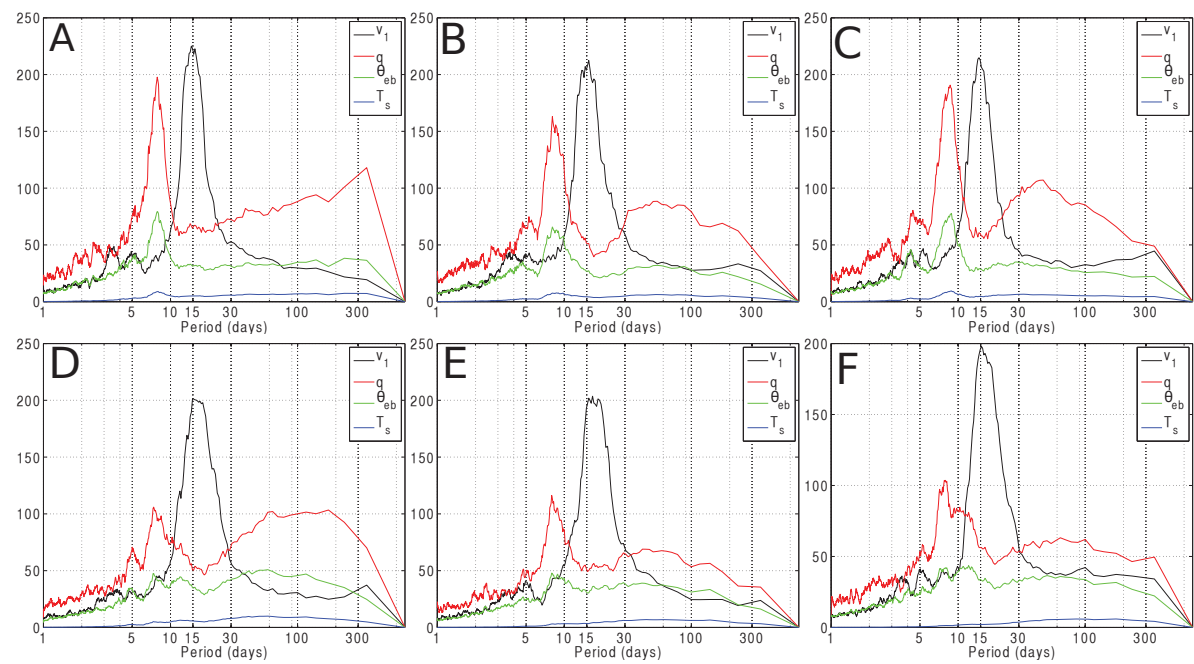

Figure 8: Power spectrum of velocity (black), moisture (red), $\theta_{e b}$ (green), and sea surface temperature (blue) from 500 days of observations at 20 equally spaced sites in the warm pool for simulations associated with a) FMK13, b) FMK13 with weak CRF ( $\alpha=0.1)$, c) FMK13 with strong CRF ( $\alpha=0.4)$, d) FMK13 with 10 meter ocean, e) FMK13 with 20 meter ocean, f) FMK13 with 40 meter ocean. Note that a slightly different $y$-axis range is used in panel (f). 
4.2. Combining effects of cloud radiative feedback and atmosphere- ocean coupling

Here we consider three distinct model CRF/AOC configurations :FMK13, FMK13 with ocean coupling, FMK13 with ocean coupling and weak $\alpha=0.1$ puted from 2000 days of data. We note that in all cases, ocean layer decreases the strength of the mean and make for more first baroclinic mode dominated circulation. The radiative convective feedback allows for slightly stronger circulation with higher second baroclinic component. For warm pool simulations, 540 radiative feedback. In addition, we use warm pool strength of $5 \mathrm{~K}$ (typical of multicloud models) and $2.5 \mathrm{~K}$ for each of three model configurations. Thus in total we consider six total parameter regimes, which highlight the combined effects of CRF and AOC, as well as SST strength.

To begin, we consider in the detail the dynamics of the spatially extended model with weak CRF and 40 meter ocean layer coupling in Figure 9. The contours of velocity field (upper left panel) show three large convective events in the center of the warm pool occurring in span of 50 days. The events are associated with build up of boundary layer moisture (lower left panel) which in turn appears to be coupled with the ocean temperature (middle left panel). These large convectively coupled waves have a high deep convective heating coupling and moisture content. They also produce faster convectively coupled gravity waves outside of the warm pool. These waves carry less moisture (lower right panel) but still produce intermittent deep convective events in the suppressed regions of the Walker circulation. We also observe a myriad of small amplitude standing wave activity in $\theta_{e b}$ and $T_{S}$ fields inside of the warm pool. These are associated with the congestus clouds that are responsible for the moistening of the mid-tropospehere and preconditioning the system for the next convective event. The congestus heating is generally abundant inside of the positive SST area of the warm pool.

The Figure 10 show mean zonal vertical structure for all the regimes comintroduction of CRF increases strength and mean of the Walker cell by about 10 percent. Unsurprisingly, weaker SST gradient results in weaker mean circula- 
tion. The effects of ocean coupling are also less drastic in the weak SST gradient

case. In fact, the simulation with ocean and CRF mechanism is stronger than the FMK regime for 2.5K SST forcing.

545 More importantly, the weaker SST gradient fundamentally changes the frequency of the generation of the synoptic scale waves inside the warm pool. Figure 11 shows deviations from zonal mean velocity field for all the regimes. The weaker $2.5 \mathrm{~K}$ warm pool shifts the variability towards 30 day convectively coupled waves which have moisture and ABL temperature spectral peak near 150 days. Unlike the mean, the amplitude of the waves is not impacted by weaker SST gradient. The simulations with ocean and CRF mechanisms appear more intermittent compared to FMK13 simulations. A remnant of the low frequency envelope can be see in the ocean coupled simulations of panel B. This pattern is hard to discern visually when CRF is added in panel C.

555 In order to elucidate the low frequency dynamics in the system, we consider the Fourier spectrum for velocity, atmospheric moisture, ABL and sea surface temperature in Figure 12. For both weak and strong SST gradient, the addition of ocean shifts the power spectrum towards the seasonal time scales. The results are mostly seen in $\mathrm{ABL}$ and atmospheric moisture while changes in velocity spectrum (as well as precipitation) are more subtle. Conversely, the introduction of the CRF shifts the spectrum towards marginally higher frequencies (moisture spectrum in particular). The weaker SST gradient fundamentally alters the moisture spectrum: from bimodal to a single low frequency peak. This change in the moisture power spectrum likely indicates slower moisture build up inside 565 of the warm pool and is responsible for the doubling of the period of generation of the synoptic scale disturbances inside of the warm pool. 

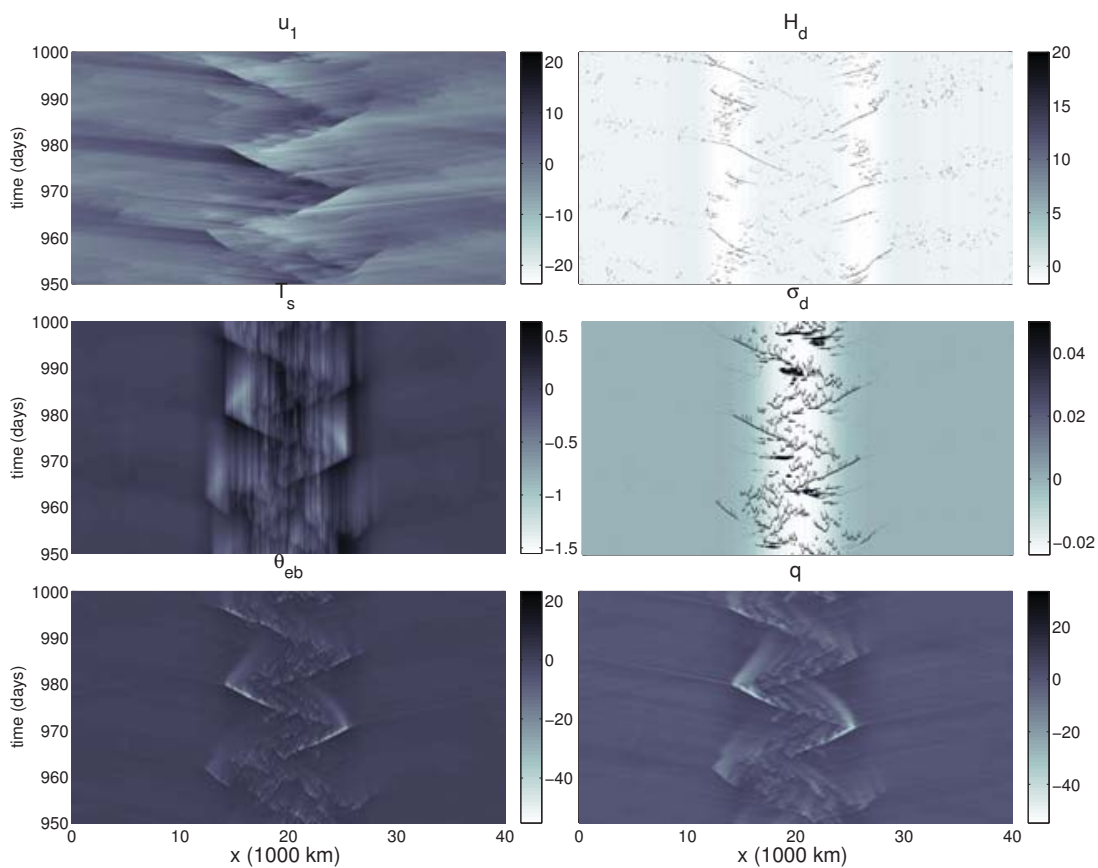

Figure 9: Hovmoller diagram showing 50 days of anomalies for stochastic multicloud model simulation with weak $\mathrm{CRF}$ and 40 meter deep ocean layer. The units of the variables are $u_{1}$ $[\mathrm{m} / \mathrm{s}], H_{d}[\mathrm{~K} / \mathrm{d}], T_{s}[\mathrm{~K}], \sigma_{d}[$ unitless $], \theta_{e b}[\mathrm{~K}], q[\mathrm{~K}]$. 


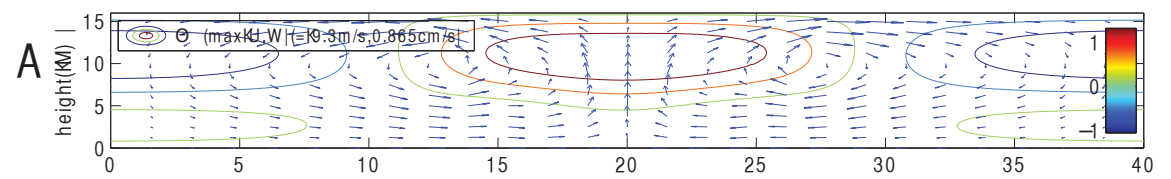

B

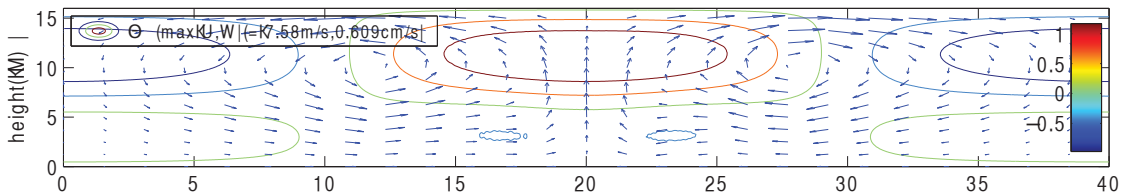

C
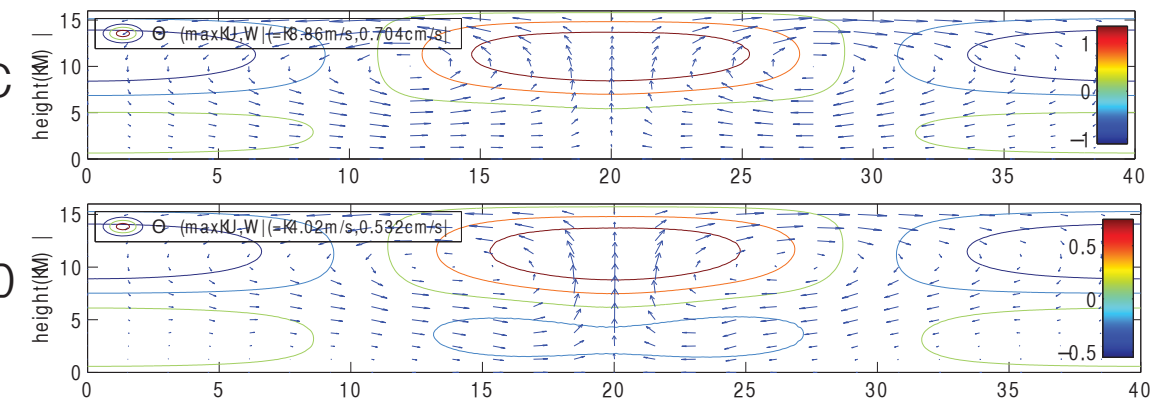

$\mathrm{E}$
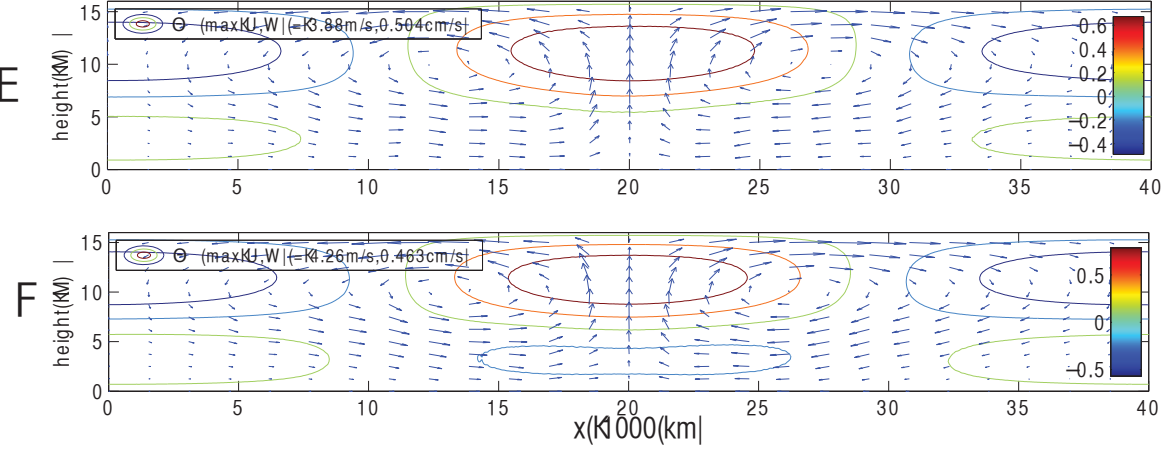

Figure 10: Mean zonal-vertical structure associated with: A) FMK13 (5K warm pool), B) FMK13 with ocean coupling (5K warm pool), C) FMK13 with ocean coupling and radiative convective feedback (5K warm pool), D) FMK13 (2.5K warm pool), E) FMK13 with ocean coupling (2.5K warm pool), F) FMK13 with ocean coupling and radiative convective feedback (2.5K warm pool). Wherever appropriate, we use strong CRF and 20 meter ocean. 

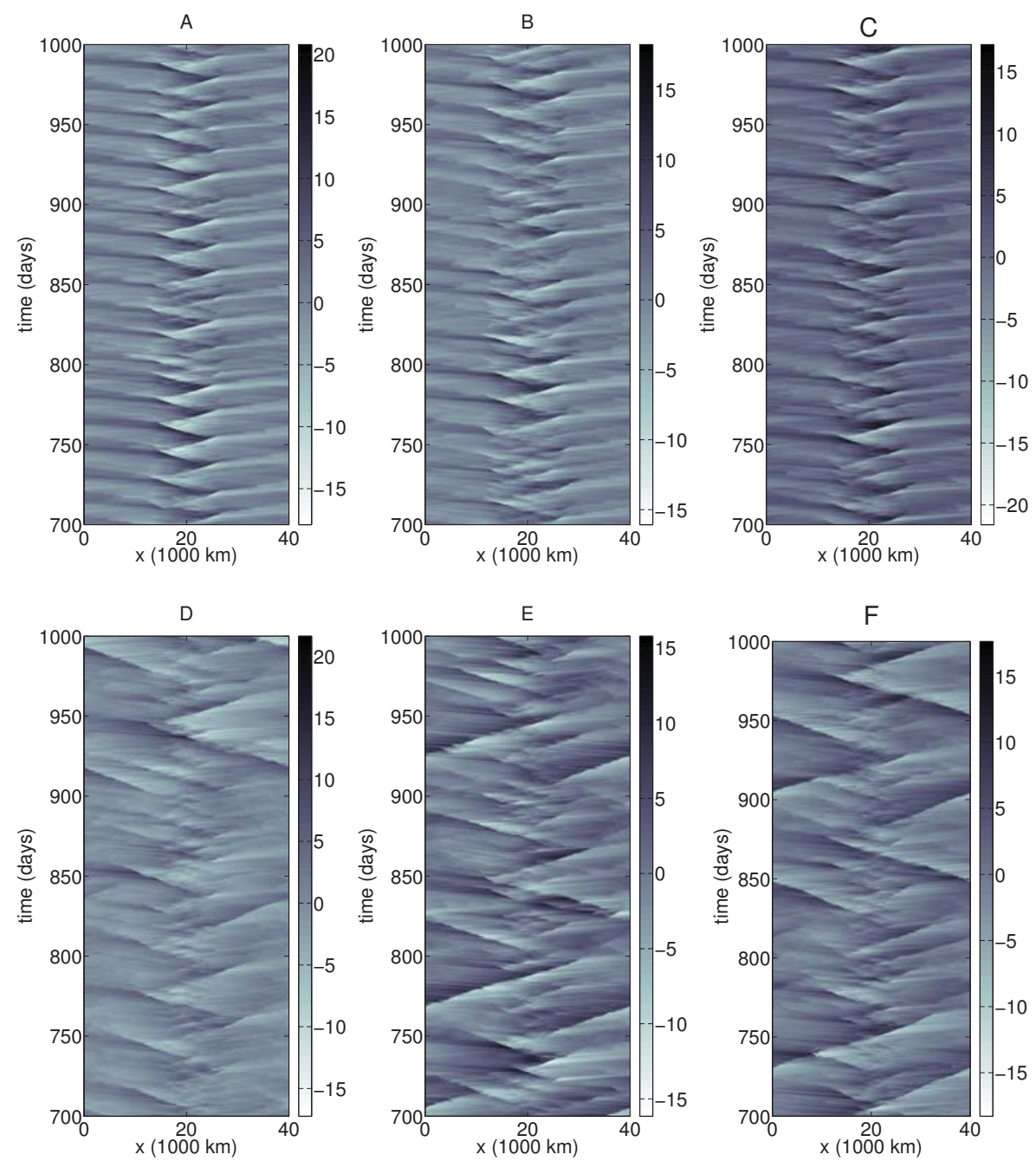

Figure 11: Contours of velocity anomalies $u_{1}(x, t)$ for an interval of 300 days for: A) FMK13 (5K warm pool), B) FMK13 with ocean coupling (5K warm pool), C) FMK13 with ocean coupling and radiative convective feedback (5K warm pool), D) FMK13 (2.5K warm pool), E) FMK13 with ocean coupling (2.5K warm pool), F) FMK13 with ocean coupling and radiative convective feedback (2.5K warm pool). Wherever appropriate, we use strong CRF and 20 meter ocean. Anomalies are computed as deviations from the time-averaged mean state. 

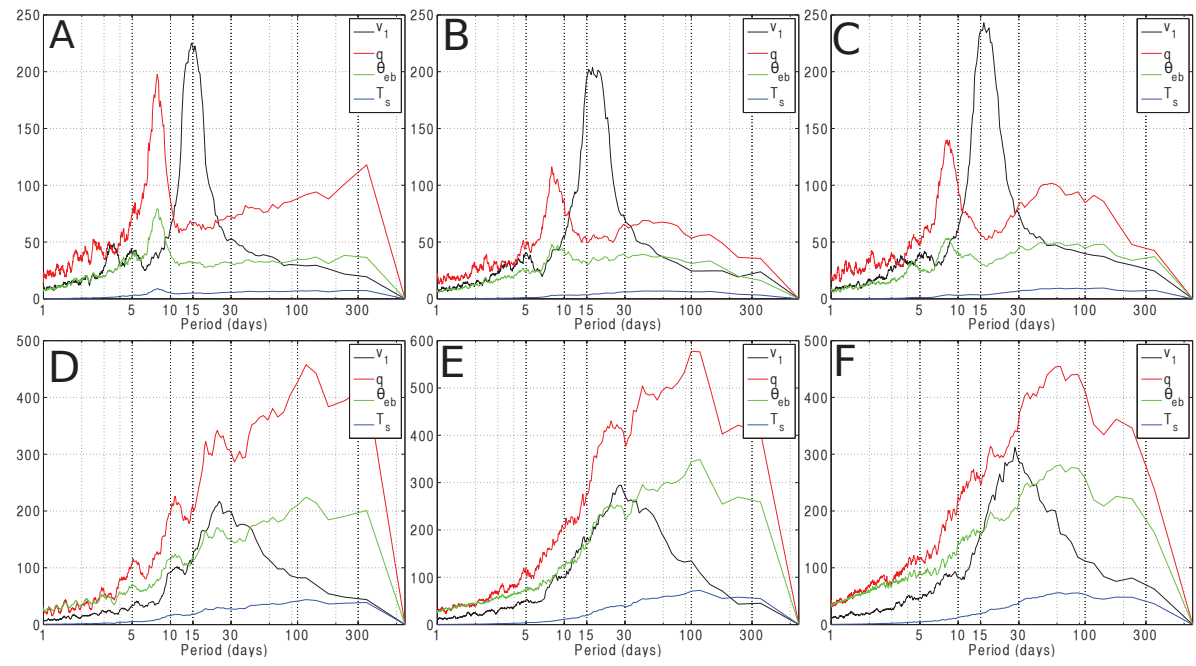

Figure 12: Power spectrum of velocity (black), moisture (red), $\theta_{e b}$ (green), and sea surface temperature (blue) from 500 days of observations at 20 equally spaced sites in the warm pool for simulations associated with: A) FMK13 (5K warm pool), B) FMK13 with ocean coupling (5K warm pool), C) FMK13 with ocean coupling and radiative convective feedback and $5 \mathrm{~K}$ warm pool, D) FMK13 (2.5K warm pool), E) FMK13 with ocean coupling and 2.5K warm pool, F) FMK13 with ocean coupling and radiative convective feedback $2.5 \mathrm{~K}$ warm pool. Wherever appropriate, we use strong CRF and 20 meter ocean. 


\section{Conclusions}

Here, the stochastic multicloud model modified with new parameterized cloud radiation feedback and atmosphere-ocean coupling mechanisms is used to 
small cloud coverage and intermittent deep convection. Cloud-radiation feedback introduces a further stochastic element to the model, as the cloud fractions are governed by a stochastic birth-death process (coupled with the large scale variables), and it thereby contributes to higher frequency variability.

In spatially extended simulations of Section 4 , the radiative convective feedback mechanism is shown to increase the mean and variability of Walker circulation. This increase in the variability is associated with low frequency convective features such as propagating synoptic scale systems originating inside of the enhanced SST area. These are similar to the cycles observed in CRM simulations (Slawinska et al., 2014b) but at a very small fraction of the computational cost. We use Fourier spectrum analysis to show that the ocean layer depth as well as the strength of the imposed SST gradient can be use to control the frequency of these low frequency convective features.

The study illustrates both the usefulness and limitation of the column model simulations for testing the mechanisms. Column simulations are essential in exploring parameter space and finding some of the catastrophic instabilities. However, the column simulations can easily exaggerate the impact of the studied mechanisms. In particular, it is clear that the intermediate and low frequency time scale features seen in the column simulations are distorted and to some extent neutralized by fast mixing by the horizontally propagating gravity waves in the spatially extended simulations.

Overall, the study develops and tests a systematic framework for incorporating parameterized radiative cloud feedback and ocean coupling. The two mechanisms are shown here to improve the representation of intraseasonal and seasonal variability. This new variability has a subtle effect on the mean state and precipitation statistics and has a great potential for interaction with other low frequency phenomena. These schemes can readily be implemented in deterministic and stochastic multicloud model GCM simulations (Khouider et al., 625 2011; Deng et al., 2014; Khouider et al., 2011; Ajayamohan et al., 2013, 2014), which already capture essential low frequency features, such as the MJO. 
Acknowledgments. The research of S.N.S is partially supported by grants NSF DMS-1209409, ONR YIP N00014-12-1-0744, and ONR MURI N00014-12-10912. The research of A. J. M. is partially supported by National Science Foundation grants DMS-0456713, DMS-1025468 and by the office of Naval Research grants ONR DRI N0014-10-1-0554 and N00014-11-1-0306 and MURI award grant ONR-MURI N-000-1412-10912. Y. F. is a postdoctoral fellow supported through A.J.Ms above NSF and ONR grants.

\section{Appendix A. Evaporative cooling at the sea surface}

We recall that evaporation term $E$, discussed in Section 2.4 is given by

$$
E=\frac{1}{\tau_{e}}\left(\theta_{e b}^{*}\left(T_{s}\right)-\theta_{e b}\right)
$$

where $\tau_{e}$ is evaporative time scale and $\theta_{e b}$ is the boundary layer equivalent potential temperature, and $\theta_{e b}^{*}$ is the saturation boundary layer equivalent potential temperature given as a function of SST. According to Claussius-Clayeypron equation, the saturation equivalent potential equivalent temperature has form

$$
\theta_{e b}^{*}=\theta_{b} \exp \left(\frac{L_{v} q^{*}\left(T_{b}\right)}{c_{p} T_{b}}\right)
$$

Here $T_{b}$ is the boundary layer temperature, $\theta_{b}$ is the boundary layer potential temperature, $L_{v}$ is the latent heat of vaporization, $q^{*}$ is the saturation specific humidity, and $c_{p}$ is the heat capacity of dry air. Although $L_{v}$ depends on temperature and $c_{p}$ depends on moisture content, the variations are small and we assume that both are constants given in Table 1.

In (A.2), $q^{*}(T)$ is the saturation specific humidity, which for an ideal gas is given by

$$
q^{*}=\frac{e^{*}}{e^{*}+\frac{R_{v}}{R_{d}}\left(p-e^{*}\right)}
$$

Here $R_{v}$ is the gas constant for water vapor, $R_{d}$ is the gas constant for dry air, $p$ is the pressure, and $e^{*}$ is the saturation water vapor pressure. I will 
take $R_{v}$ and $R_{d}$ to have the constant values given in Table 1 . I will also take $p=p_{s}=1000 \mathrm{hPa}$, since the latent heat exchange takes place at the ocean surface. If we assume that $L_{v}$ is constant, then the equation (A.3) can be integrated to give the following equation for $e^{*}$ :

$$
e^{*}(T)=e^{*}\left(T_{r e f}\right) \exp \left(\frac{L_{v}}{R_{v}}\left[\frac{1}{T_{r e f}}-\frac{1}{T}\right]\right)
$$

We therefore have $\theta_{e b}^{*}$ as a function of $T$ by using (A.2), (A.3), and (A.4). We approximate $T_{b}$ and $\theta_{b}$ in these equations with the SST $T_{s}$, and we assume $L_{v}$ and $c_{p}$ take the constant values listed in Table 1. (A.2), (A.3), and (A.4) take the form

$$
\begin{gathered}
\theta_{e b}^{*}=\theta_{b} \exp \left(\frac{L_{v} q^{*}\left(T_{s}\right)}{c_{p} T_{s}}\right) \\
q^{*}\left(T_{s}\right)=\frac{e^{*}\left(T_{s}\right)}{e^{*}\left(T_{s}\right)+\frac{R_{v}}{R_{d}}\left(p-e^{*}\left(T_{s}\right)\right)} \\
e^{*}\left(T_{s}\right)=e^{*}\left(T_{r e f}\right) \exp \left(\frac{L_{v}}{R_{v}}\left[\frac{1}{T_{r e f}}-\frac{1}{T_{s}}\right]\right)
\end{gathered}
$$

The equations (A.5), (A.6), and (A.7) show nonlinear relationship between $\theta e b^{*}$ and $T_{s}$. It appears that $\theta e b^{*}$ rises by $5 \mathrm{~K}$ for $1 \mathrm{~K}$ rise in $T_{s}$, in the range $20-35$ $\mathrm{K}$ as seen in Figure A.13 ( taking $T_{r e f}=301 \mathrm{~K}$,). A lfinear approximation of this nonlinear relationship below

$$
\theta_{e b}^{*} \approx 5 T_{s}
$$

is used in the model computations.

\section{References}

Ajayamohan, R. S., Khouider, B., Majda, A. J., 2013. Realistic initiation and dynamics of the Madden-Julian Oscillation in a coarse resolution aquaplanet GCM. Geophys. Res. Lett. 40 (23), 6252-6257.

URL http://dx.doi.org/10.1002/2013GL058187 
Ajayamohan, R. S., Khouider, B., Majda, A. J., 2014. Simulation of monsoon intraseasonal oscillations in a coarse-resolution aquaplanet GCM. Geophys. Res. Lett. 41 (15), 5662-5669.

Bony, S., Emanuel, K. A., 2005. On the Role of Moist Processes in Tropical Intraseasonal Variability: Cloud-Radiation and Moisture-Convection Feedbacks. Journal of Atmospheric Sciences 62, 2770-2789.

Cess, R. D., Potter, G. L., Zhang, M.-H., Blanchet, J.-P., Chalita, S., Colman, R., Dazlich, D. A., del Genio, A. D., Dymnikov, V., Galin, V., Jerrett, D., Keup, E., Lacis, A. A., Le Treut, H., Liang, X.-Z., Mahfouf, J.-F., McAvaney, B. J., Meleshko, V. P., Mitchell, J. F. B., Morcrette, J.-J., Norris, P. M., Randall, D. A., Rikus, L., Roeckner, E., Royer, J.-F., Schlese, U., Sheinin, D. A., Slingo, J. M., Sokolov, A. P., Taylor, K. E., Washington, W. M., Wetherald, R. T., Yagai, I., 1990. Intercomparison and interpretation of climate feedback processes in 19 atmospheric general circulation models. Geophysical Research 95, 601-615.

Cess, R. D., Zhang, M. H., Ingram, W. J., Potter, G. L., Alekseev, V., Barker, H. W., Cohen-Solal, E., Colman, R. A., Dazlich, D. A., Del Genio, A. D., Dix, M. R., Dymnikov, V., Esch, M., Fowler, L. D., Fraser, J. R., Galin, V., Gates, W. L., Hack, J. J., Kiehl, J. T., Le Treut, H., Lo, K. K.-W., McAvaney, B. J., Meleshko, V. P., Morcrette, J.-J., Randall, D. A., Roeckner, E., Royer, J.-F., Schlesinger, M. E., Sporyshev, P. V., Timbal, B., Volodin, E. M., Taylor, K. E., Wang, W., Wetherald, R. T., 1996. Cloud feedback in atmospheric general circulation models: An update. Journal of Geophysical Research 101, 12791.

Cox, S. K., Griffith, K., 1979a. Estimates of Radiative Divergence during Phase III of the GARP Atlantic Tropical Experiment Part I. Methodology. Journal of Atmospheric Sciences 35, 576-585.

${ }_{695}$ Cox, S. K., Griffith, K., 1979b. Estimates of Radiative Divergence during Phase 
III of the GARP Atlantic Tropical Experiment: Part II. Analysis of Phase III Results. Journal of Atmospheric Sciences 36, 586-601.

Deng, Q., Khouider, B., Majda, A. J., 2014. The MJO in a coarse-resolution gcm with a stochastic multicloud parameterization. J. Atmos. Sci., in press.

ECMWF, 2003. Proceedings ECMWF/CLIVAR Workshop on Simulation and Prediction of Intraseasonal variability with Emphasis on the MJO, 3-6 November.

Frenkel, Y., Khouider, B., Majda, A. J., 2010. Simple multicloud models for the diurnal cycle of tropical precipitation. Part I: Formulation and the case of the tropical oceans. J. Atmos. Sci. 68 (10), 2192-2207.

Frenkel, Y., Majda, A. J., Khouider, B., 2012. Using the stochastic multicloud model to improve tropical convective parameterization: A paradigm example. J. Atmos. Sci. 69 (3), 1080-1105.

Frenkel, Y., Majda, A. J., Khouider, B., 2013. Stochastic and deterministic multicloud parameterizations for tropical convection. Climate Dynamics 41, $1527-1551$.

Gillespie, D. T., 1975. An exact method for numerically simulating the stochastic coalescence process in a cloud. J. Atmos. Sci. 32, 1977-1989.

Gillespie, D. T., 1977. Exact stochastic simulation of coupled chemical reactions. The Journal of Physical Chemistry 81 (25), 2340-2361.

Grabowski, W. W., 2001. Coupling cloud processes with the large-scale dynamics using the cloud-resolving convection parameterization. J. Atmos. Sci. 58 (9), 978-997.

Grabowski, W. W., 2004. An improved framework for superparameterization. 720 J. Atmos. Sci. 61 (15), 1940-1952. 
Grabowski, W. W., Smolarkiewicz, P. K., 1999. CRCP: A Cloud Resolving Convection Parameterization for modeling the tropical convecting atmosphere. Physica D Nonlinear Phenomena 133, 171-178.

Han, Y., Khouider, B., 2010. Convectively coupled waves in a sheared environment. J. Atmos. Sci. 67, 2913-2942.

Hendon, H. H., Liebmann, B., 1994. Organization of convection within the Madden-Julian oscillation. J. GEOPHYS. RES. 99, 8073-8084.

Johnson, R. H., Rickenbach, T. M., Rutledge, S. A., Ciesielski, P. E., Schubert, W. H., 1999. Trimodal characteristics of tropical convection. Journal of Climate 12 (8), 2397-2418.

Katsoulakis, M. A., Majda, A. J., Vlachos, D. G., 2003. Coarse-grained stochastic processes for microscopic lattice systems. Proceedings of the National Academy of Sciences of the United States of America 100 (3), 782-787.

Katsoulakis, M. A., Majda, A. J., Vlachos, D. G., 2003b. Coarse-grained stochastic processes and monte carlo simulations in lattice systems. Journal of Computational Physics 186 (1), 250 - 278.

Khouider, B., 2014. A coarse grained stochastic multi-type particle interacting model for tropical convection: Nearest neighbour interactions. Comm. Math. Sci 12, 1379-1407.

Khouider, B., Biello, j., Majda, A. J., 2010. A stochastic multicloud model for tropical convection. Comm. Math. Sci. 8 (1), 187-216.

Khouider, B., Majda, A. J., 2005a. A non oscillatory balanced scheme for an idealized tropical climate model; Part I: Algorithm and validation. Theoretical and Computational Fluid Dyn. 19, 331-354.

745 Khouider, B., Majda, A. J., 2005b. A non oscillatory balanced scheme for an idealized tropical climate model; Part II: Nonlinear coupling and moisture effects. Theoretical and Computational Fluid Dyn. 19, 355-375. 
Khouider, B., Majda, A. J., 2006a. Multicloud convective parametrizations with crude vertical structure. Theor. Comp. Fluid Dyn. 20, 351-375.

Khouider, B., Majda, A. J., 2006b. A simple multicloud parametrization for convectively coupled tropical waves. Part I: Linear analysis. J. Atmos. Sci. $63,1308-1323$.

Khouider, B., Majda, A. J., 2007. A simple multicloud parametrization for convectively coupled tropical waves. Part II: Nonlinear simulations. J. Atmos. Sci. $64,381-400$.

Khouider, B., Majda, A. J., 2008a. Equatorial convectively coupled waves in a simple multicloud model. J. Atmos. Sci. 65, 3376-3397.

Khouider, B., Majda, A. J., 2008b. Multicloud models for organized tropical convection: Enhanced congestus heating. J. Atmos. Sci. 65, 897-914.

Khouider, B., Majda, A. J., Katsoulakis, M. A., 2003. Coarse-grained stochastic models for tropical convection and climate. Proceedings of the National Academy of Science 100, 11941-11946.

Khouider, B., St-Cyr, A., Majda, A. J., Tribbia, J., 2011. The MJO and convectively coupled waves in a coarse-resolution GCM with a simple multicloud parameterization. J. Atmos. Sci. 68 (2), 240-264.

URL http://dx.doi.org/10.1175/2010JAS3443.1

Kiehl, J. T., 1993. On the Observed Near Cancellation between Longwave and Shortwave Cloud Forcing in Tropical Regions. J. Climate 7, 559-565.

Kikuchi, K., Wang, B., 2008. Diurnal precipitation regimes in the global tropics. J. Climate 21, 2680-2696.

Lau, W. K. M., Waliser, D. E., 2005. Intraseasonal Variability in the Atmosphere-Ocean Climate System. Springer-Verlag.

Lin, J.-L., Kiladis, G. N., Mapes, B. E., Weickmann, K. M., Sperber, K. R., Lin, W., Wheeler, M. C., Schubert, S. D., Del Genio, A., Donner, L. J., Emori, 
S., Gueremy, J.-F., Hourdin, F., Rasch, P. J., Roeckner, E., Scinocca, J. F., 2006. Tropical intraseasonal variability in 14 IPCC AR4 climate models. Part I: Convective signals. Journal of Climate 19 (12), 2665-2690.

Lindzen, R. S., Nigam, S., 1987. On the role of sea surface temperature gradients in forcing low-level winds and convergence in the tropics. J. Atmos. Sci. 44 (17), 2418-2436.

Majda, A. J., 2007. Multiscale models with moisture and systematic strategies for superparameterization. J. Atmos. Sci. 64 (7), 2726-2734.

Majda, A. J., Franzke, C., Khouider, B., 2008. An applied mathematics perspective on stochastic modelling for climate. Philosophical Transactions of the Royal Society A: Mathematical, Physical and Engineering Sciences 366 (1875), 2427-2453.

Majda, A. J., Shefter, M. G., 2001. Models for Stratiform Instability and Convectively Coupled Waves. Journal of Atmospheric Sciences 58, 1567-1584.

Majda, A. J., Stechmann, S., Khouider, B., 2007. MaddenJulian Oscillation analog and intraseasonal variability in a multicloud model above the equator. Proceedings of the National Academy of Science 104, 9919-9924.

Mapes, B. E., 2000. Convective inhibition, subgrid-scale triggering energy, and stratiform instability in a toy tropical wave model. J. Atmos. Sci. 57 (10), $1515-1535$.

Moncrieff, M., Shapiro, M., Slingo, J., Molteni, F., 2007. Collaborative research at the intersection of weather and climate. WMO Bulletin 56, 204-211.

Moncrieff, M. W., Klinker, E., 1997. Organized convective systems in the tropical western Pacific as a process in general circulation models: A TOGA COARE case-study. Quarterly Journal of the Royal Meteorological Society $123,805-827$. 
Nakazawa, T., 1974. Tropical super clusters within intraseasonal variation over the western pacific. J. Meteorol. Soc. Japan 66, 823-839.

Peters, K., Jakob, C., Davies, L., Khouider, B., Majda, A. J., 2013. Stochastic behavior of tropical convection in observations and a multicloud model. J. $805 \quad$ Atmos. Sci. 70, 3556- 3575.

Peters, M. E., Bretherton, B., 2005. A Simplified Model of the Walker Circulation with an Interactive Ocean Mixed Layer and Cloud-Radiative Feedbacks. Journal of Climate 18, 4216-4234.

Randall, D., Khairoutdinov, M., Arakawa, A., Grabowski, W., 2003. Breaking the cloud parameterization deadlock. Bulletin of the American Meteorological Society 84 (11), 1547-1564.

Schumacher, C., Houze, R. A., Kraucunas, I., 2004. The tropical dynamical response to latent heating estimates derived from the TRMM Precipitation Radar. Journal of Atmospheric Sciences 61, 1341-1358.

Scinocca, J. F., McFarlane, N. A., 2004. The variability of modeled tropical precipitation. J. Atmos. Sci. 61, 1993-2015.

Slawinska, J., Pauluis, O., Majda, A. J., Grabowski, W. W., 2014a. Multi-scale interactions in an idealized walker circulation: Simulations with sparse spacetime superparameterization. Mon Weather Review in press.

Slawinska, J., Pauluis, O., Majda, A. J., Grabowski, W. W., 2014b. Multiscale Interactions in an Idealized Walker Circulation: Mean Circulation and Intraseasonal Variability. Journal of Atmospheric Sciences 71, 953-971.

Slingo, J. M., Sperber, K. R., Boyle, J. S., Ceron, J., Dix, M., Dugas, B., Ebisuzaki, W., Fyfe, J., Gregory, D., Gueremy, J., Hack, J., Harzallah, A., ${ }_{825}$ Inness, P., Kitoh, A., Lau, W., McAvaney, B., Madden, R., Matthews, A., Palmer, T. N., Parkas, C., Randall, D., Renno, N., 1996. Intraseasonal oscillations in 15 atmospheric general circulation models: results from an AMIP diagnostic subproject. Climate Dynamics 12, 325-357. 
Sobel, A. H., Bretherton, C. S., Gildor, H., Peters, M. E., 2004. Convection, cloud-radiative feedbacks and thermodynamic ocean coupling in simple models of the walker circulation. In: Wang, C., Xie, S. P., Carton, J. A. (Eds.), Earth's Climate. American Geophysical Union, pp. 393-405.

Stephens, G., Webster, P., 1979. Sensitivity of radiative forcing to variable cloud and moisture. J. Atmos. Sc. 36, 1542-1556.

Takayabu, Y. N., 1994. Large-scale cloud disturbances associated with equatorial waves. part I: Spectral features of the cloud disturbances. J. Meteor. Soc. Japan 72, 433-448.

Tian, B., Ramanathan, V., 2003. A simple moist tropical atmosphere model: The role of cloud radiative forcing. Journal of Climate 16 (12), 2086-2092.

Wheeler, M., Kiladis, G. N., 1999. Convectively coupled equatorial waves: Analysis of clouds and temperature in the wavenumber-frequency domain. J. Atmos. Sci. 56 (3), 374-399.

Wu, X., Moncrief, M. W., 2000. Long-Term Behavior of Cloud Systems in TOGA COARE and Their Interactions with Radiative and Surface Processes: Effects on the Energy Budget and SST. Journal of Atmospheric Sciences 58, $1155-1167$.

Xing, Y., Majda, A. J., Grabowski, W. W., 2009. New efficient sparse spacetime algorithms for superparameterization on mesoscales. Monthly Weather Review 137 (12), 4307-4324.

Zhang, C., 2005. Madden-Julian oscillation. Reviews of Geophysics 43, RG2003.

Zurovac-Jevtik, D., Bony, S., Emanuel, K. A., 2005. On the Role of Clouds and Moisture in Tropical Waves: A Two-Dimensional Model Study. Journal of Atmospheric Sciences 63, 2140-2155. 


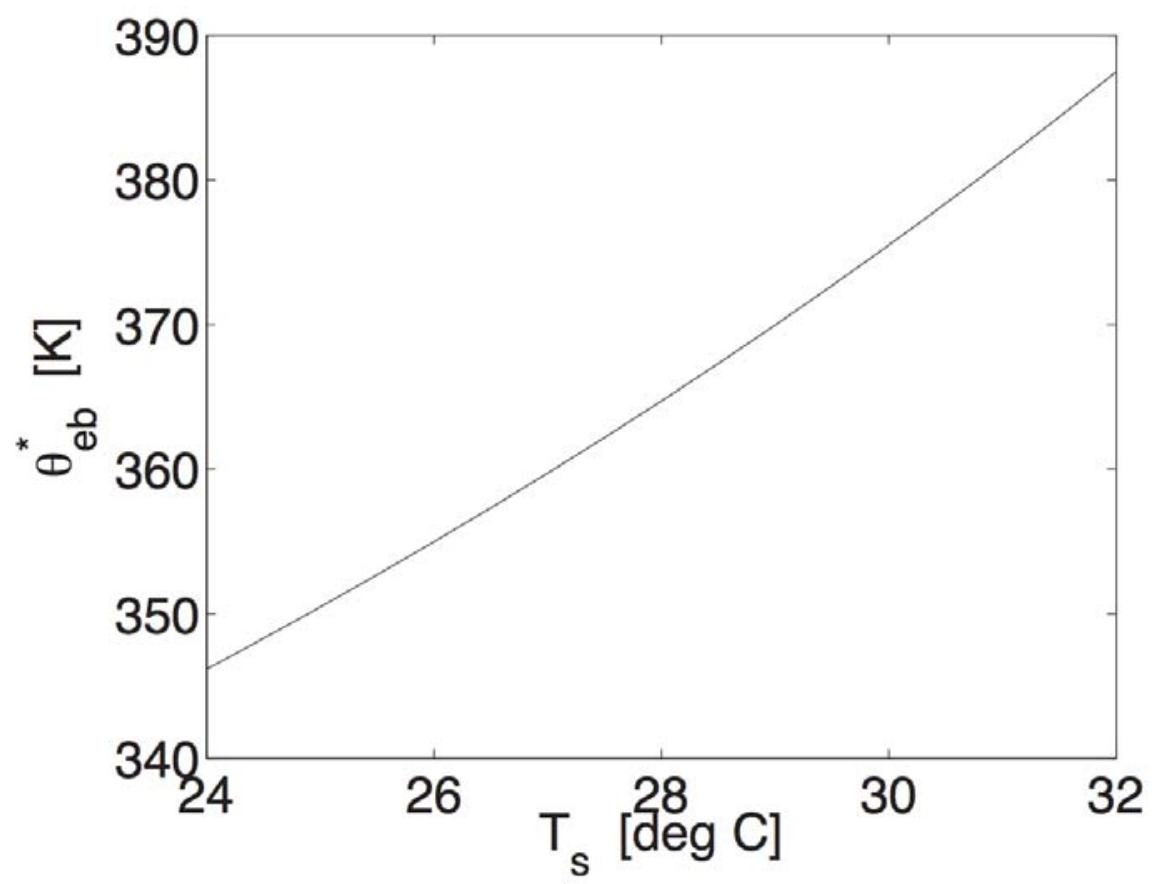

Figure A.13: The nonlinear relationship between $\theta e b^{*}$ and $T_{s}$. It appears that $\theta e b^{*}$ rises by $5 \mathrm{~K}$ for $1 \mathrm{~K}$ rise in $T_{s}$. A linear approximation of this relationship is use in the model computations. 